\title{
Cardiac hypertrophy and arrhythmia in mice induced by a mutation in ryanodine receptor 2
}

\author{
Francisco J. Alvarado, ${ }^{1}$ J. Martijn Bos, ${ }^{2,3}$ Zhiguang Yuchi, ${ }^{4}$ Carmen R. Valdivia, ${ }^{1}$ \\ Jonathan J. Hernández, ${ }^{5}$ Yan-Ting Zhao, ${ }^{6}$ Dawn S. Henderlong, ${ }^{7}$ Yan Chen, ${ }^{7}$ Talia R. Booher, ${ }^{1}$ \\ Cherisse A. Marcou, ${ }^{3}$ Filip Van Petegem, ${ }^{8}$ Michael J. Ackerman, ${ }^{2,3,9}$ and Héctor H. Valdivia ${ }^{1}$ \\ 'Department of Medicine, Division of Cardiovascular Medicine, and Cardiovascular Research Center, University of \\ Wisconsin-Madison School of Medicine and Public Health, Madison, Wisconsin, USA. ${ }^{2}$ Department of Pediatric and \\ Adolescent Medicine, Division of Pediatric Cardiology, and ${ }^{3}$ Department of Molecular Pharmacology and Experimental \\ Therapeutics, Windland Smith Rice Sudden Death Genomic Laboratory, Mayo Clinic, Rochester, Minnesota, USA. \\ ${ }^{4}$ Tianjin Key Laboratory for Modern Drug Delivery \& High-Efficiency, Collaborative Innovation Center of Chemical Science \\ and Engineering, School of Pharmaceutical Science and Technology, Tianjin University, Tianjin, China. ${ }^{5}$ Department \\ of Pediatrics, University of Wisconsin-Madison School of Medicine and Public Health, Madison, Wisconsin, USA. \\ ${ }^{6}$ Department of Pharmacology and 'Department of Internal Medicine, University of Michigan, Ann Arbor, Michigan, USA. \\ ${ }^{8}$ Department of Biochemistry and Molecular Biology, University of British Columbia, Vancouver, British Columbia, Canada. \\ ${ }^{9}$ Department of Cardiovascular Medicine, Division of Heart Rhythm Services, Mayo Clinic, Rochester, Minnesota, USA..
}

Hypertrophic cardiomyopathy (HCM) is triggered mainly by mutations in genes encoding sarcomeric proteins, but a significant proportion of patients lack a genetic diagnosis. We identified a potentially novel mutation in ryanodine receptor 2, RyR2-P1124L, in a patient from a genotypenegative HCM cohort. The aim of this study was to determine whether RyR2-P1124L triggers functional and structural alterations in isolated RyR2 channels and whole hearts. We found that P1124L induces significant conformational changes in the SPRY2 domain of RyR2. Recombinant RyR2-P1124L channels displayed a cytosolic loss-of-function phenotype, which contrasted with a higher sensitivity to luminal $\left[\mathrm{Ca}^{2+}\right]$, indicating a luminal gain of function. Homozygous mice for RyR2-P1124L showed mild cardiac hypertrophy, similar to the human patient. This phenotype, evident at 1 year of age, was accompanied by an increase in the expression of calmodulin (CaM). P1124L mice also showed higher susceptibility to arrhythmia at 8 months of age, before the onset of hypertrophy. RyR2-P1124L has a distinct cytosolic loss-of-function and a luminal gain-of-function phenotype. This bifunctionally divergent behavior triggers arrhythmias and structural cardiac remodeling, and it involves overexpression of CaM as a potential hypertrophic mediator. This study is relevant to continue elucidating the possible causes of genotype-negative HCM and the role of RyR2 in cardiac hypertrophy.

Conflict of interest: MJA is a consultant for Audentes Therapeutics, Boston Scientific, Gilead Sciences, Invitae, Medtronic, MyoKardia, and St. Jude Medical. MJA and Mayo Clinic have an equity/ royalty relationship with Alive Cor, Blue $0 x$ Health, and StemoniX but without remuneration thus far.

Copyright: (c) 2019 American Society for Clinical Investigation

Submitted: December 13, 2018 Accepted: February 26, 2019 Published: April 4, 2019.

Reference information: /Cl Insight. 2019;4(7):e126544. https://doi. org/10.1172/jii.insight.126544.

\section{Introduction}

Hypertrophic cardiomyopathy (HCM) is one of the most common congenital cardiac diseases, affecting approximately 1 out of every 500 individuals ( $0.2 \%$ of the general population) (1). HCM is characterized at the tissue level by myocyte hypertrophy and disarray, as well as interstitial fibrosis. Clinical features involve thickening of the LV wall, diastolic dysfunction, heart failure, and increased risk of life-threatening arrhythmias that may lead to sudden cardiac death (SCD) (2, 3). Mutations affecting over 24 genes have been implicated in the pathogenesis of $\operatorname{HCM}(4,5)$, most commonly encoding sarcomeric proteins. For example, mutations in the genes $M Y H 7$ and $M Y P B C 3$, encoding myosin heavy chain 7 and cardiac myosin-binding protein C (MyBP-C), respectively, account for approximately $90 \%$ of patients with HCM who have a positive genetic test result (6). However, approximately half of the patients who meet the diagnostic criteria of HCM and undergo genetic testing are negative for mutations in the genes included in HCM panels $(4,7)$. As genetic testing has become more common and gene panels expand, new HCM-associated mutations have been identified in several genes encoding the myofilaments and beyond. While rare, 
there are reports suggesting that mutations in genes encoding $\mathrm{Ca}^{2+}$-handling proteins contribute to $\mathrm{HCM}$ pathogenesis, including genes such as sorcin ( $R R I)$, calsequestrin 2 (CASQ2), troponin $\mathrm{C} 1$ (TNNC1), and ryanodine receptor 2 (RYR2) (8). Except for a limited number of TNNC1 mutations (9), HCM associated to perturbations in $\mathrm{Ca}^{2+}$-handling proteins has not been characterized thoroughly.

RyR2 is the major $\mathrm{Ca}^{2+}$ release channel in the heart and localizes on the sarcoplasmic reticulum (SR). During an action potential, a small inward $\mathrm{Ca}^{2+}$ current through L-type $\mathrm{Ca}^{2+}$ channels activates RyR2, triggering more $\mathrm{Ca}^{2+}$ release from the SR. RyR2 provides most of the $\mathrm{Ca}^{2+}$ required for this process, called excitation-contraction (e-c) coupling. Tight regulation of $\mathrm{Ca}^{2+}$ release in the heart, and thus of RyR2 function, is essential because the same $\mathrm{Ca}^{2+}$ used for e-c coupling can induce $\mathrm{Ca}^{2+}$-dependent arrhythmias (10) or modulate hypertrophic signaling pathways (11). Over the last 20 years, nearly 200 RyR2 mutations have been identified in patients with catecholaminergic polymorphic ventricular tachycardia (CPVT) (12), a disorder involving severe ventricular arrhythmias triggered by stress but without structural alterations of the myocardium. The mechanisms underlying RyR2-mediated CPVT (CPVT1) have been the subject of extensive research, uncovering a variety of molecular and cellular mechanisms for $\mathrm{Ca}^{2+}$ mishandling and, ultimately, arrhythmia. More recently, a RyR2 mutation, T1107M, was reported in a family with HCM (13). This mutation generated considerable interest in the field, as it was the first RyR2 variant potentially contributing to a structural disorder (HCM) rather than an entirely functional disorder (CPVT). Recombinant A1107M, the mouse analog, behaved considerably different in a heterologous expression system when compared with other CPVT1 mutations, suggesting a different pathogenic mechanism (14). T1107M has also appeared in cohorts of whole-exome sequencing (WES) (15) and of CPVT patients (16), weakening its potential as an HCM-associated mutation. Altogether, these data indicate that RyR2 can produce a wide range of cardiac dysfunctions, but the link between RyR2 mutations and structural cardiomyopathy remains elusive.

In this study, we describe the comprehensive characterization of a potentially novel RyR2 variant (P1124L), identified in a patient with sarcomere mutation-negative HCM. While the P1124L variant is in the same domain as $\mathrm{T} 1107 \mathrm{M}$, herein we present defining characteristics of $\mathrm{P} 1124 \mathrm{~L}$ that bolster its potential association with the patient's phenotype, including (a) satisfying a genetic criterion (identification of a rare variant in RYR2 in a patient with HCM), (b) in silico tools suggesting pathogenicity, (c) molecular and functional criteria (validation assays demonstrating that this variant is disruptive functionally), and (d) whole organism/animal model criterion (mice harboring P1124L develop arrhythmia and hypertrophy, although not as dramatically as the patient). Hearts from P1124L mice showed an increase in the expression of calmodulin (CaM), a classical inhibitor of RyR2 and signaling molecule but had no significant changes in the expression of downstream signaling molecules involved in hypertrophy. Thus, although the precise molecular mechanisms underlying hypertrophy in P1124L mice could not be fully discerned and need further study, here we offer evidence that links RyR2 dysfunction with cardiac hypertrophic remodeling, in addition to the demonstrated role of this protein in the pathogenicity in CPVT.

\section{Results}

Identification of RyR2-P1124L in a human patient with HCM. Among the 36 patients with genotype-negative/ phenotype-positive HCM who underwent WES, in 1 of them we identified a potentially novel, candidate HCM-associated variant in RYR2, P1124L, without any other candidate disease-associated mutations. This RyR2-P1124L-positive patient was a male diagnosed with HCM at 17 years of age following a syncopal episode and was placed on $\beta$-blocker therapy. Four months later, because of persistent chest tightness and paroxysmal nocturnal dyspnea, he received a dual chamber pacemaker elsewhere. He was evaluated at Mayo Clinic 1 year later, exhibiting moderate concentric hypertrophy $(21 \mathrm{~mm})$ in the absence of hypertension (Supplemental Figure 1; supplemental material available online with this article; https://doi.org/10.1172/ jci.insight.126544DS1). The ejection fraction (EF) was 75\% with decreased left ventricle (LV) chamber size and hyperdynamic function. Obstruction of the LV outflow tract (LVOT) was dynamic, measuring $46 \mathrm{mmHg}$ at rest and increasing to $88 \mathrm{mmHg}$ after provocation. There was also evidence of systolic anterior motion of the mitral valve with grade III/IV mitral regurgitation. ECG monitoring showed artifacts consistent with a rhythm maintained by firing of the pacemaker, but without signs of ventricular arrhythmia. Because the symptoms remained refractory to pharmacotherapy and pacing, the patient underwent an extensive surgical myectomy at Mayo Clinic. An ECG performed 4 days after surgery showed normal sinus rhythm, signs of left atrial enlargement, LV hypertrophy, and QRS widening. Pathological examination of the surgically 
removed myocardium showed mild endocardial thickening, severe myocyte hypertrophy, mild interstitial fibrosis, and myocyte disarray.

No HCM-causative mutations were noted in any of the established HCM-susceptibility genes, including all the canonical genes implicated in sarcomeric HCM: MYBPC3, MYH7, TNNT2, TNNI3, TNNC1, TPM1, ACTC, MYL2, and MYL3. Instead, a base change was identified following WES in RYR2, generating the missense variant RyR2-P1124L. To our knowledge, this variant has never been associated with CPVT1 and is extremely rare, being found in 5 of 246,156 alleles in the Genome Aggregation Database (gnomAD) giving it a minor allele frequency of 0.00002 (http://gnomad.broadinstitute.org/). Additionally, consensus results from the most commonly used in silico tool for variant prediction suggested P1124 is likely pathogenic or damaging (7 of 7 tools used; CADD-score, 32; PolyPhen-2, probably damaging; SIFT/ PROVEAN, deleterious; PANTHER, probably damaging; AlignGVD, most likely damaging [C65]; MutationTaster, probably damaging; Grantham score, 98). At the time of his evaluation at Mayo Clinic, the patient's mother had no evidence for HCM, while the father and 3 siblings were not screened; nonetheless, the patient reported a positive family history of HCM in 2 maternal cousins. Secondary to familial nonparticipation, additional evidence of cosegregation could not be determined. Given that there are not multiple unrelated cases with putative RyR2-mediated HCM, without compelling functional data, $R Y R 2$ in general and RyR2-P1124L, in particular, would be classified as a gene of uncertain significance (GUS) and variant of uncertain significance (VUS; RyR2-P1124L-American College of Medical Genetics [RyR2-P1124LACMG] pathogenic supporting criteria: PP3 [computational evidence supporting deleterious effect] and PP4 [phenotype is specific for a disease with a single genetic etiology]), respectively, in terms of an association with HCM in accordance with the 2015 ACMG criteria for variant interpretation (17). To determine the potential association between P1124L and HCM, we studied this mutation on multiple levels.

Localization of P1124L in the 3-dimensional structure of RyR2. CPVT1-associated RyR2 mutations mainly cluster in 4 hot spots, which coincide with functional domains of the channel, called CPVT-I to -IV (Figure 1A). P1124, a highly conserved residue among species (Figure 1B) and RyR isoforms (Supplemental Figure 2), is located outside these hot spots in SPRY2, one of the 3 SPRY domains of RyR2 (Figure 1C). The crystal structures of SPRY1 and SPRY2 were solved recently to a resolution below $1.5 \AA(18,19)$, while high-resolution structures of SPRY3 are not available. P1124 is part of a flexible linker connecting strands $\beta 2$ and $\beta 3$ that was not included in the initial SPRY2 model due to poor electron density; however, recent cryo-electron microscopy (cryo-EM) structures of RyR1 and RyR2 suggest that this loop is at the SPRY2SPRY3 interface (Figure 1D) (20, 21).

Since all RyR cryo-EM structures originate from native material, analyzing RyR disease mutants is currently not feasible. We therefore crystallized the SPRY2 domain containing the P1124L mutation and solved its structure at an average $1.44 \AA$ resolution. The loop containing the mutation is well ordered, in contrast to the WT, which shows less-defined density (19). The WT loop could still be traced; thus, we further refined this structure to allow a direct comparison (Supplemental Figure 3, Supplemental Figure 4, and Supplemental Table 1). Introducing the proline $(\mathrm{P})$ to leucine $(\mathrm{L})$ substitution produces an extensive conformational change that affects neighboring residues, notably C1122 and D1125, with D1125 swinging outside and facing SPRY3 (Figure 1, E and F, and Supplemental Figure 4). Also, a new salt bridge forms between R1119 and D1132, located in opposite ends of the $\beta 2-\beta 3$ linker. Overall, these changes provide stability to the $\beta 2-\beta 3$ linker, explaining why the density for this loop is better defined in the P1124L mutant. Details of the SPRY2-SPRY3 interactions cannot yet be discerned in the cryo-EM reconstructions because the local resolution in those areas is low. However, just like mutations analyzed in the $\mathrm{N}$-terminal disease hot spot, such changes at interdomain interfaces are likely to cause relative domain orientations that indirectly affect channel gating $(22,23)$. For example, the altered D1125 side chain may affect interactions with R1599 in SPRY3, although this remains to be tested.

Molecular phenotype of P1124L-containing RyR2 channels. To determine the possible molecular alterations induced by $\mathrm{P} 1124 \mathrm{~L}$ in RyR2, we introduced the mutation into the mouse Ryr2 cDNA and expressed the WT or P1124L protein in HEK293 cells. The approach is justified because P1124 and the neighboring region of SPRY2 are highly conserved among species (Figure 1B and Supplemental Figure 2). Indeed, within the entire SPRY2 domain, mouse and human RyR2 protein only differ in the 3 residues highlighted in Figure 1B. Forty-eight hours after transfection, cell lysates were used for functional studies. We found that expression of P1124L-containing RyR2 channels was significantly reduced compared with the WT RyR2 (Supplemental Figure 5A). This is an uncommon observation (reported only for 1 CPVT-associated mutation; ref. 24), as mutation-harboring RyR2 channels are often expressed to the same level as WT channels in this 


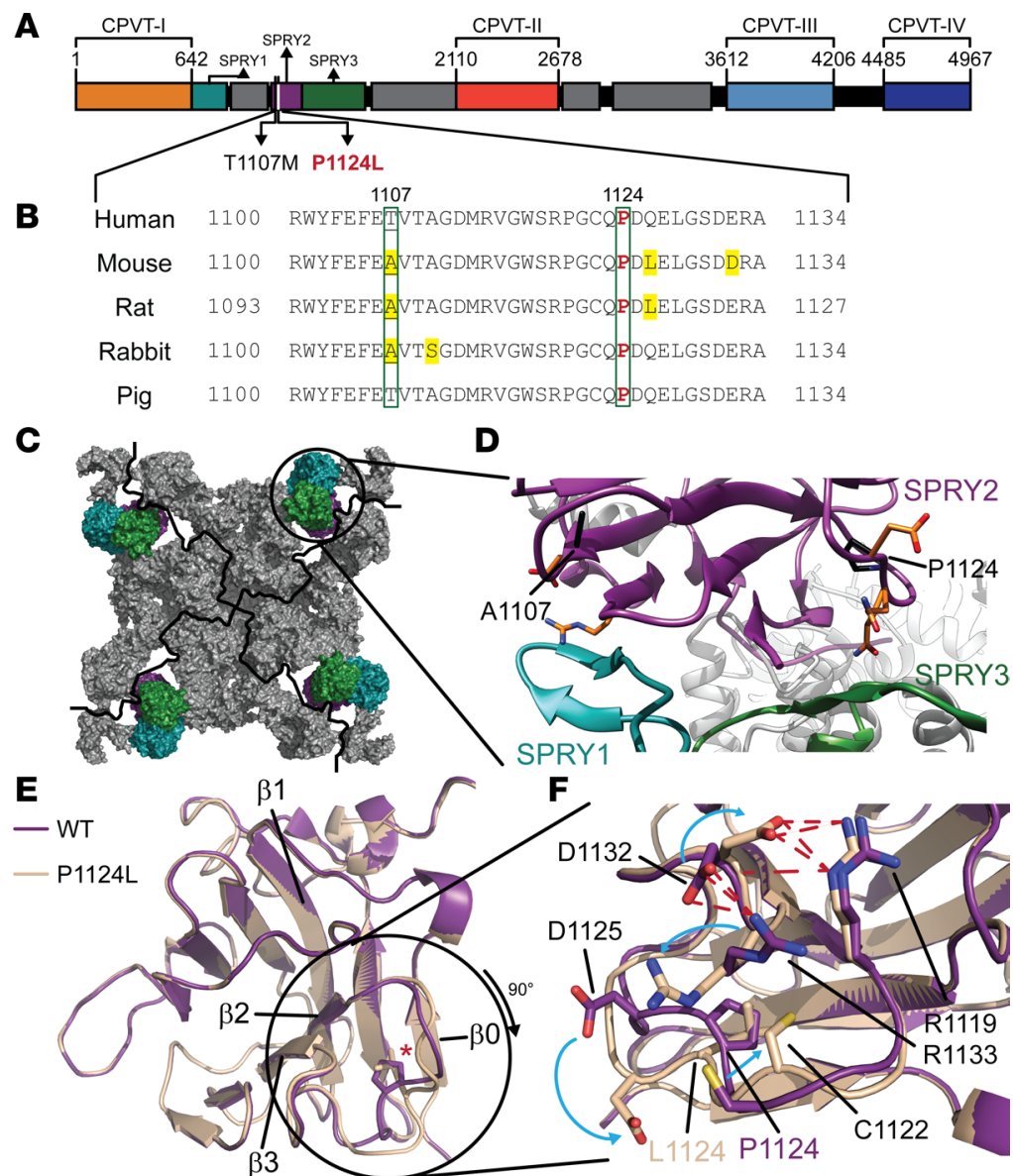

Figure 1. Position of P1124L in RyR2 and crystal structure of the SPRY2 domain. (A) Linear representation of a single ryanodine receptor 2 (RyR2) subunit, indicating the regions where CPVT mutations are clustered (CPVT-I through -IV). The 3 SPRY domains are indicated and color-coded in all panels. (B) Amino acid sequence of a region of SPRY2 RyR2 from 5 relevant species. Residues corresponding to the human 1107 and 1124 are highlighted. Accession numbers and other species and isoforms are indicated in Supplemental Figure 2. (C) Electron density map of RyR2 from the literature (20). Subunits are delimited by black lines. (D) Modeled interface between the 3 SPRY domains of RyR2. The positions of the 2 HCM-associated mutations, A1107 and P1124, are indicated. (E and F) Superimposed crystal structures of the WT and P1124L SPRY2 domains obtained from the literature (19) and this work, respectively. Asterisk indicates positions of residue 1124 within the $\beta 2-\beta 3$ linker. Blue arrows indicate movement of 4 residues between WT and P1124L structures. Red dashed lines indicate salt bridges.

heterologous system (14). Next, we performed $\left[{ }^{3} \mathrm{H}\right]$ ryanodine binding assays to assess RyR2 channel activity. Ryanodine only binds to the open state of the channel; hence, $\left[{ }^{3} \mathrm{H}\right]$ ryanodine binding is suitable to evaluate RyR2 activity in a population of channels at varying cytosolic $\left[\mathrm{Ca}^{2+}\right]$. To account for the different RyR2 density between groups, we performed Western blots to determine the expression level in each transfection as a percentage of the WT samples (Supplemental Figure 5A); $\left[{ }^{3} \mathrm{H}\right]$ ryanodine binding was then normalized to this value. We found that $\mathrm{P} 1124 \mathrm{~L}$ behaves as a loss-of-function mutation, with lower maximum $\left.{ }^{3} \mathrm{H}\right] \mathrm{ryan}-$ odine binding than WT (Figure 2, A and B) and requiring higher $\left[\mathrm{Ca}^{2+}\right]$ for activation (Figure 2, C and D).

Single channel recordings. Recombinant RyR2 channels from heterologous systems are expressed in the absence of other important regulatory cofactors. Therefore, we prepared SR-enriched microsomal fractions from pooled WT or homozygous (Homo) P1124L mouse hearts to assess RyR2 activity from native channels reconstituted in planar lipid bilayers (the strategy used to create mice harboring P1124L is described in the following sections). Figure 2, E and F, shows representative current traces and histograms obtained from single RyR2 channels from SR microsomes. P1124L channels showed lower open probability than WT channels (Figure $2 \mathrm{G}$ ) at nominally free $\left[\mathrm{Ca}^{2+}\right](3-5 \mu \mathrm{M})$, while average unitary current at $0 \mathrm{mV}$ in a $\mathrm{Cs}^{+}$gradient was not altered (trans/cis 300/50 mM; Figure $2 \mathrm{H}$ ). These data agree with the $\left[{ }^{3} \mathrm{H}\right]$ ryanodine binding assays and support the idea that $\mathrm{P} 1124 \mathrm{~L}$ is a loss-of-function mutation, at least at physiologically relevant cytosolic $\left[\mathrm{Ca}^{2+}\right]$. 

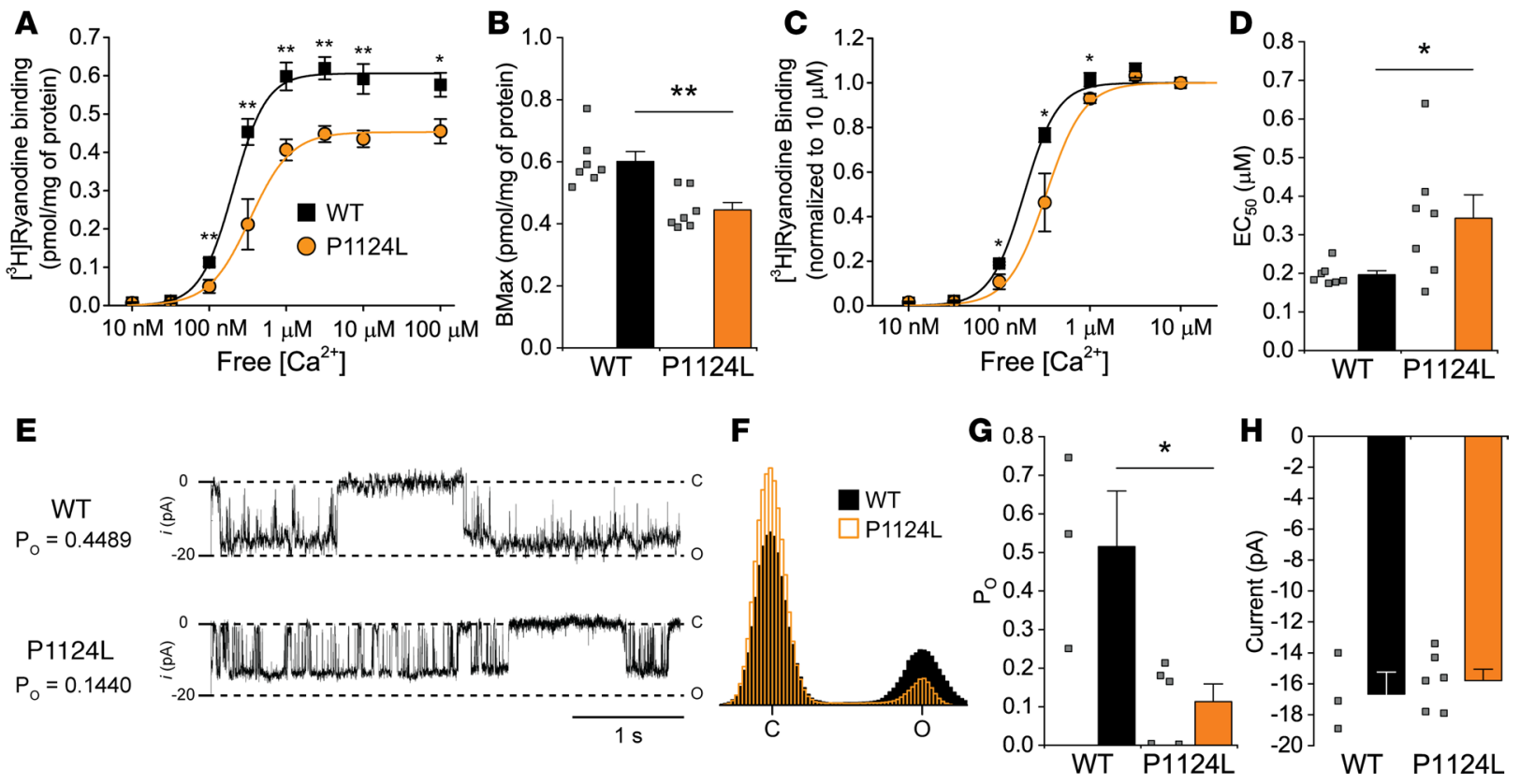

Figure 2. Molecular phenotype of RyR2-P1124L. (A) $\mathrm{Ca}^{2+}$-dependent $\left[{ }^{3} \mathrm{H}\right]$ ryanodine binding curve corrected for ryanodine receptor 2 (RyR2) expression. The mouse Ryr2 cDNA was transiently transfected into HEK293 cells. Cells were lysed 48 hours after transfection. Recombinant RyR2 was then incubated with $\left[{ }^{3} \mathrm{H}\right]$ ryanodine in the presence of increasing concentrations of $\left[\mathrm{Ca}^{2+}\right]$ to activate the channel. $\left.{ }^{3} \mathrm{H}\right]$ ryanodine was determined by liquid scintillation. (B) Maximum binding $\left(B_{\text {Max }}\right)$ calculated from the curves in $A$ using Hill's equation. (C) $\mathrm{Ca}^{2+}$-dependent $\left[{ }^{3} \mathrm{H}\right]$ ryanodine binding curves (same as in $\mathbf{A}$ ) normalized to 10 $\mu \mathrm{M}\left[\mathrm{Ca}^{2+}\right]$. (D) $\mathrm{EC}_{50}$ for $\left[\mathrm{Ca}^{2+}\right]$-dependent $\left[{ }^{3} \mathrm{H}\right]$ ryanodine binding $\left(\mathbf{A}-\mathbf{D}, n=7\right.$ curves from at least 4 independent transfections. ${ }^{*} P<0.05$, ${ }^{* *} P<0.01$, rank sum test). (E) Representative single RyR2 channel recordings from cardiac microsomes prepared WT and homozygous (Homo) P1124L hearts. Channels were fused into artificial planar lipid bilayers. Single channel current and open probability $\left(P_{0}\right)$ were recorded at nominally free $\left[\mathrm{Ca}^{2+}\right]$ in a $300 / 50 \mathrm{mM}\left[\mathrm{s}^{+}\right.$gradient. (F) Overlapped histograms of normalized current calculated from 2 representative channels. (C) Average $P_{0}$ of WT and P1124L channels. (H) Average single channel current calculated from recordings in a 300/50 mM Cs ${ }^{+}$gradient ( $G$ and $\mathbf{H}, n=3$ WT, 5-6 P1124L channels. ${ }^{*} P<0.05$, 2-tailed $t$ test).

Spontaneous $\mathrm{Ca}^{2+}$ oscillations in HEK293 cells expressing RyR2. Using heterologous expression of RyR2 in HEK293 cells and monitoring of intra-ER $\left[\mathrm{Ca}^{2+}\right]$, Tang et al. (14) determined that some RyR2 mutations induce abnormal termination of spontaneous $\mathrm{Ca}^{2+}$ release (SCR) events triggered by ER $\mathrm{Ca}^{2+}$ overload. We used a similar approach to gain a better understanding of the possible mechanisms of dysfunction associated with P1124L. Inducible HEK293 cells stably expressing RyR2 were transfected with the fluorescence resonance energy transfer-based (FRET-based) ER Ca ${ }^{2+}$ sensor D1ER (25). Then, cells were perfused with $2 \mathrm{mM} \mathrm{Ca}^{2+}$ to fill the ER and induce luminal $\left[\mathrm{Ca}^{2+}\right]$ oscillations due to spontaneous RyR2 activation. Under these conditions, oscillations are observed as a decrease in the FRET ratio. Figure 3A shows representative FRET traces measured in cells expressing WT and P1124L channels. From these recordings, we determined the FRET level at which $\mathrm{Ca}^{2+}$ oscillations activate $\left(\mathrm{F}_{\text {Act }}\right)$ and terminate $\left(\mathrm{F}_{\mathrm{Term}}\right)$. Tetracaine $(1 \mathrm{mM})$ was perfused to inhibit RyR2 and determine the FRET level at maximum store capacity $\left(\mathrm{F}_{\mathrm{Max}}\right)$, followed by $20 \mathrm{mM}$ caffeine to empty the ER and determine the residual FRET signal $\left(\mathrm{F}_{\mathrm{Min}}\right)$. The store capacity was determined as $\mathrm{F}_{\mathrm{Max}}-\mathrm{F}_{\min }$. In both groups, the activation threshold was $\sim 80 \%$ of the maximum ER store capacity (Figure 3B); however, $\mathrm{P} 1124 \mathrm{~L}$ showed a significant reduction in the termination threshold (Figure 3C), resulting in a larger fractional release (Figure 3D). These observations did not involve a change in the maximum capacity of the ER store (Figure 3E). Interestingly, while the percentage of HEK293 cells showing oscillations of intra-ER $\left[\mathrm{Ca}^{2+}\right]$ was not different (Figure 3F), cells expressing the P1124L channel showed an increased number of oscillations over the monitoring period (Figure 3G). Similar to HEK293 cells with transient transfection of P1124L, cells with stable expression also showed lower RyR2 expression (Supplemental Figure 5B). The lower expression of P1124L channels may suggest reduced stability of the channel protein in heterologous systems, but this remains to be tested. Altogether, these data suggest that P1124L channels have impaired luminal regulation and, upon activation, may release more $\mathrm{Ca}^{2+}$ than WT channels.

Generation and cardiac structural phenotype of P1124L knock-in mice. Knock-in RyR2-P1124L mice were generated following the strategy summarized in Supplemental Figure 6A. Using homologous recombination, we introduced 3 base substitutions in exon 28 of Ryr2 (Supplemental Figure 6B): a silent A 
A
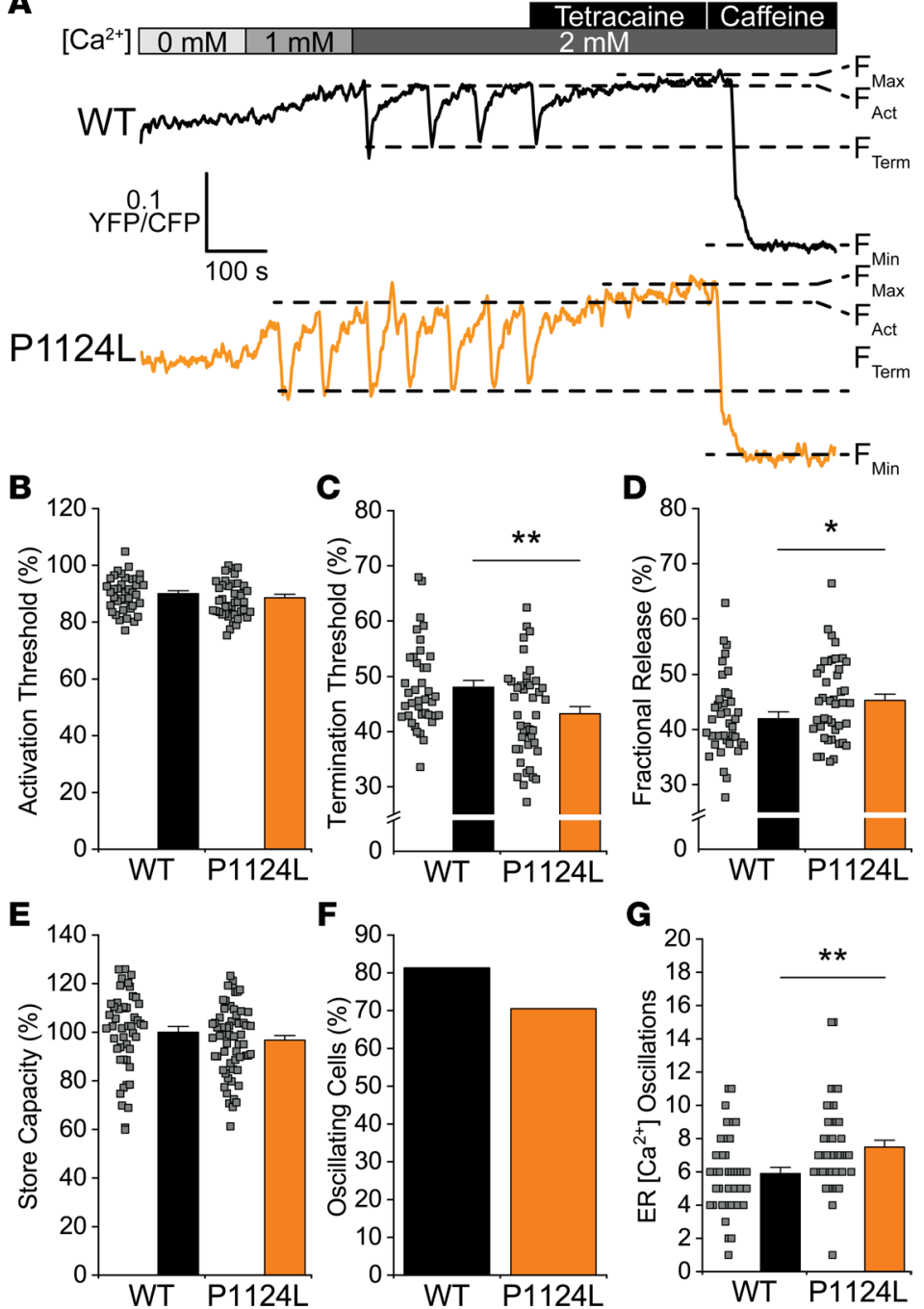

Figure 3. Abnormal termination of spontaneous $\mathrm{Ca}^{2+}$ release in HEK293 cells expressing RyR2. (A) Representative traces of the intra-ER FRET signal using the intra-ER Ca ${ }^{2+}$ sensor D1ER in cells expressing RyR2. Stable HEK293 cells with inducible expression of the mouse RyR2 were transfected with D1ER. RyR2 expression was induced with $1 \mu \mathrm{g} /$ $\mathrm{ml}$ tetracycline 18-24 hours after transfection. Time-lapse imaging of D1ER FRET was performed after 18-24 hours of RyR2 induction. Images were collected for 3 minutes at $\mathrm{O}\left[\mathrm{Ca}^{2+}\right], 3$ minutes at $1 \mathrm{mM}\left[\mathrm{Ca}^{2+}\right], 5$ minutes at $2 \mathrm{mM}$ $\left[\mathrm{Ca}^{2+}\right], 5$ minutes in the presence of $1 \mathrm{mM}$ tetracycline to inhibit RyR2 and measure maximum ER Ca $\mathrm{a}^{2+}$ capacity $\left(\mathrm{F}_{\mathrm{Max}}\right)$, and about 3 minutes in the presence of $20 \mathrm{mM}$ caffeine to empty the ER and measure the residual FRET signal $\left(F_{\text {Min }}\right)$. (B-D) Activation (B) and termination (C) thresholds and fraction of the ER Ca ${ }^{2+}$ content released (D) during spontaneous oscillations $\left({ }^{*} P<0.05,{ }^{*} P<0.01\right.$, rank sum test [B], 2-tailed $t$ test [C-D]). (E) ER store capacity determined as $\left(F_{\text {Max }}-F_{\text {Min }}\right)$ and expressed as percentage of WT (rank sum test). (F) Percentage of cells showing oscillations

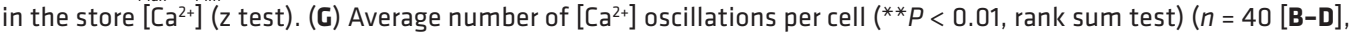
$49[\mathbf{E}], 87[\mathbf{F}]$, and $39[\mathbf{G}]$ WT cells; 44 [B-D], 61 [E], 104 [F] and 43 [G] P1124L cells from 4 independent D1ER transfections and inductions of RyR2 expression).

to $\mathrm{G}$ substitution in codon 1123, creating a PvuII restriction site, and 2 substitutions in codon 1124 , accounting for the $\mathrm{P}$ to $\mathrm{L}$ change. $\mathrm{P} 1124 \mathrm{~L}$ mice show Mendelian propagation (Supplemental Figure 6C). To determine the physiological effect of the molecular dysfunction observed in RyR2-P1124L channels, we performed a thorough characterization of the mice harboring the mutation. At 8 months of age, both Homo and heterozygous (Het) P1124L mice showed comparable cardiac structure and function with WT littermates, although Homo mice also showed significantly lower heart rate (HR) than Het mice (Supplemental Table 2). At 1 year of age, this difference in HR was no longer evident, and RyR2-P1124L mice showed mild but significant hypertrophy (Figure 4A and Supplemental Table 3). This phenotype is evident in both Homo and Het mice, and it involves thickening of the posterior wall (Figure 4B) and 
A

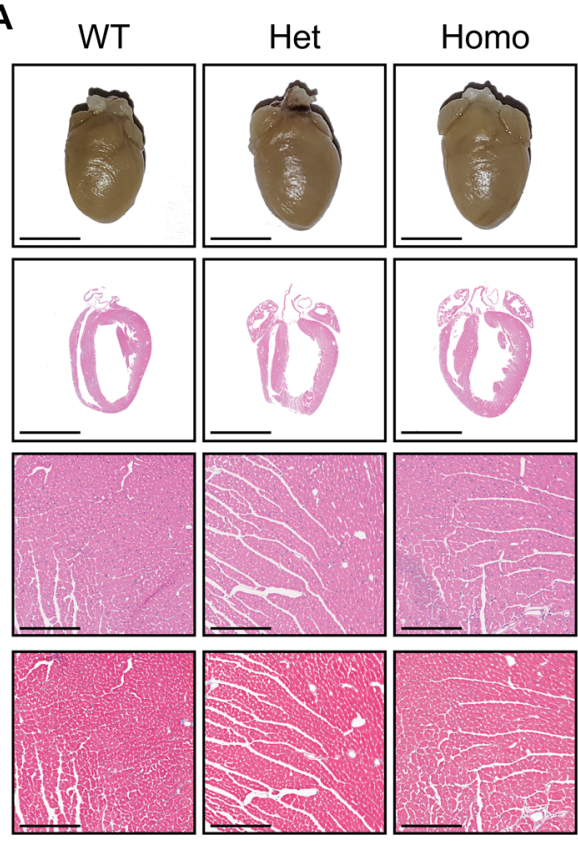

B
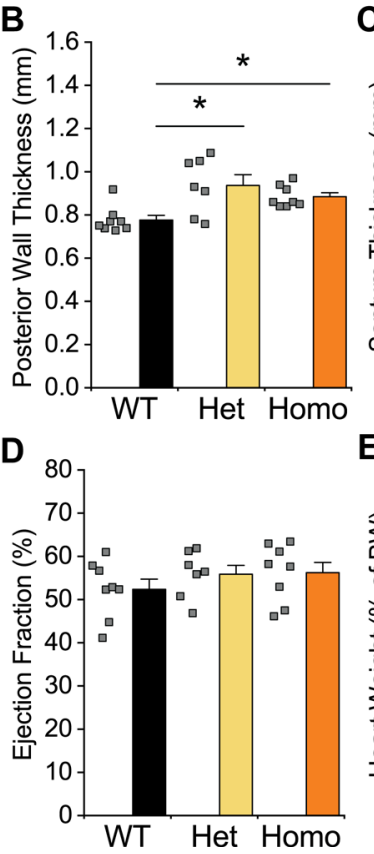

C

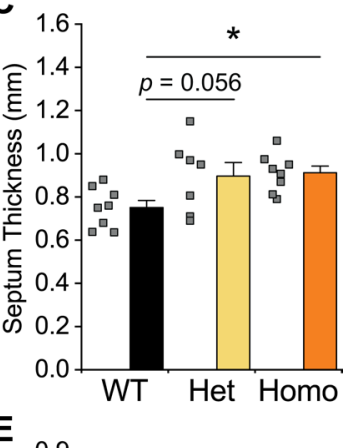

E

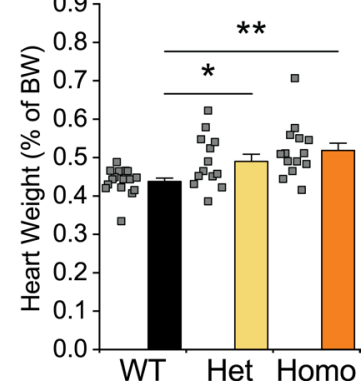

Figure 4. Cardiac hypertrophy in 1-year-old P1124L mice. (A) Representative images of formalin-fixed hearts (first-row scale bar: $5 \mathrm{~mm}$ ), H\&E-stained coronal sections at low magnification of FFPE tissue (second-row scale bar: $5 \mathrm{~mm}$ ), micrographs of H\&E-stained tissue sections (third-row scale bar: $200 \mu \mathrm{m}$ ), and micrographs of Masson's Trichromestained tissue sections (fourth-row scale bar: $200 \mu \mathrm{m}$ ). Images are representative of $n=3$ hearts per genotype. (B-D) M-Mode echocardiography measurements of posterior wall (B) and septum thickness (C), as well as ejection fraction (D). Mice were lightly anesthetized with isoflurane during the recordings. (E) Heart weight as a percentage of total BW ( $n=8$ WT, 7 Het, 8 Homo mice. ${ }^{*} P<0.05$, ANOVA on Ranks [panel B], one-way ANOVA [panel C-E]).

septum (Figure 4C), without deterioration of the EF (Figure 4D). In both Het and Homo mice, we also observed a significant increase in heart weight as percentage of BW (Figure 4E), without significant differences in BW (Supplemental Table 3). We did not observe microscopic alterations of the cardiac muscle or fibrotic infiltrations (fibrosis quantification: WT, 4.54\% $\pm 0.57 \%$; Het, $5.21 \% \pm 0.74 \%$; Homo, $4.19 \% \pm 0.57 \% ; P>0.05,1$-way ANOVA; Figure 4A).

As an independent test of hypertrophy, we measured isolated ventricular myocytes from 1-year-old P1124L mice. To avoid bias, fields of view were selected randomly to observe myocytes plated on 35-mm glass-bottom dishes. Myocytes within the field of view were centered and focused prior to collecting transmitted light images using a confocal microscope; measurements were performed offline. All the cells in an image were measured unless they were partially out of frame or out of focus (Figure 5A). While we did not detect a difference in cell width (Figure 5B), Homo myocytes were longer than WT and Het myocytes (Figure 5C), resulting in Homo myocytes having a significantly larger surface area than WT (Figure 5D). Finally, we measured the cardiac expression of 3 hypertrophic gene markers using quantitative PCR (qPCR): natriuretic peptide A (Nppa), natriuretic peptide B (Nppb), and myosin heavy chain 7 (Myh7). Consistent with the mild hypertrophy, we only measured a significant increase in Nppa expression in Homo mice (Figure 5E).

Taken together, these data suggest that $\mathrm{P} 1124 \mathrm{~L}$ induces mild cardiac hypertrophy without overt hemodynamic dysfunction in mice, similar to the patient's clinical presentation. This structural phenotype is detectable in both Homo and Het mice at 1 year of age, but the number of significant differences in indexes of hypertrophy were greater in Homo mice. We have followed P1124L mice for $\sim 70$ weeks of age ( 1.3 years) without observing evident differences in sudden death or overall mortality.

Signaling pathways associated with cardiac hypertrophy. To determine the mechanisms by which P1124L might induce hypertrophy, we used Western blots to measure the expression and phosphorylation of relevant signaling molecules. Unlike our previous observation in HEK293 cells, we did not detect differences in the expression of RyR2 in mice under 3-6 months or over 1 year of age (Figure 6, A and B). This suggests that the mechanisms reducing the expression of $\mathrm{P} 1124 \mathrm{~L}$ channels in heterologous systems are compensated for in the mouse heart. Additionally, we did not observe differences in the phosphorylation 
A
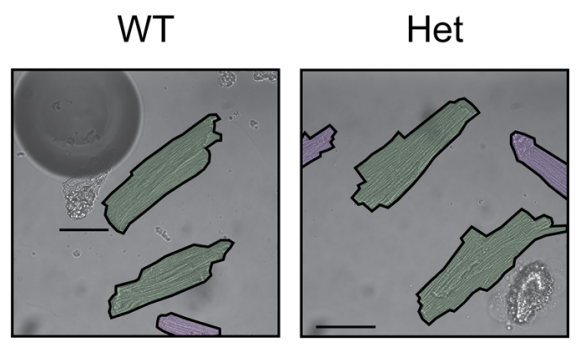

C

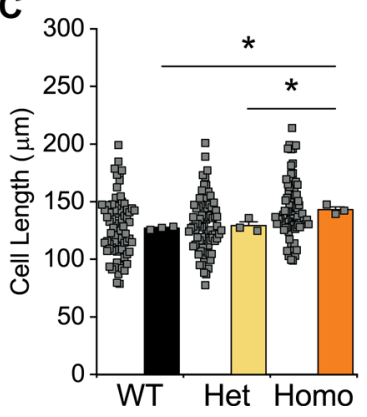

D

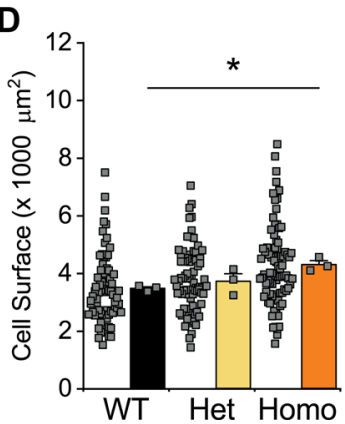

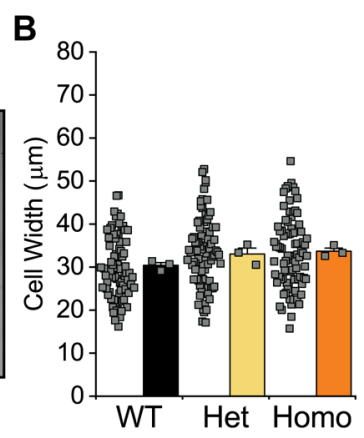

E

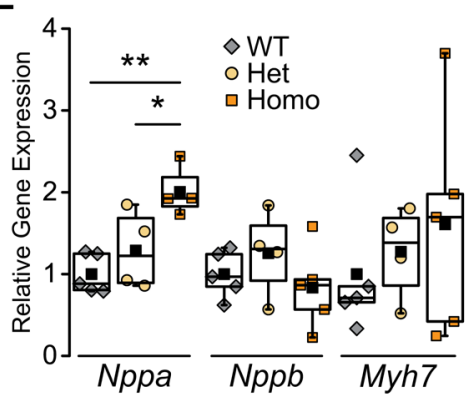

Figure 5. Cellular hypertrophy in cardiac myocytes from 1-year-old P1124L mice. (A) Representative transmitted light images of ventricular myocytes (scale bar: $50 \mu \mathrm{m}$ ). Cardiac myocytes were enzymatically isolated from freshly explanted hearts. Images were obtained with a confocal microscope. Cells are delineated and colored green (measured cells) or purple (out of focus or out of frame). (B-D) Cell width (B), length (C), and surface area (D) measured from transmitted light images (left, individual measurements from $n=75$ WT, 81 Het, 84 Homo cells; right, mean values for $n=3$ hearts per genotype. ${ }^{*} P<0.05$, 1-way ANOVA). (E) Box plots showing transcript expression level of 3 hypertrophic genes: natriuretic peptide $\mathrm{A}(\mathrm{Nppa})$, natriuretic peptide $\mathrm{B}(\mathrm{Nppb})$, and myosin heavy chain 7 (Myh7). Data normalized to actin (Actb) expression ( $n=5$ WT, 4 Het, 5 Homo hearts per genotype. ${ }^{* *} P<0.01,{ }^{* *} P<0.05,1$-way ANOVA).

level of RyR2 (Supplemental Figure 7) or the expression other e-c coupling proteins (Supplemental Figure 8) in hearts of 1-year-old mice. We observed evident variability within samples of the same genotype, and tendencies - such as an increase in NCX expression (Supplemental Figure 8C) - were not significant. Nevertheless, GAPDH expression showed little variation, suggesting that the variability in the e-c coupling proteins is not due to inadequate normalization (Supplemental Figure 8I).

We then assessed the expression of relevant molecules from $2 \mathrm{Ca}^{2+}$-dependent signaling pathways associated with cardiac hypertrophy: CaM-CaMKII-HDAC and CaM-CaN-NFAT. In both cases, CaM acts as a $\mathrm{Ca}^{2+}$ sensor that activates either the $\mathrm{Ca}^{2+} / \mathrm{CaM}$-dependent kinase II (CaMKII) or the phosphatase calcineurin $(\mathrm{CaN})$. Downstream, each pathway follows a different signaling cascade that ultimately activates hypertrophic genes (26). At 1 year of age, we did not detect a difference in the expression or autophosphorylation levels of CaMKII between genotypes (Supplemental Figure 9). We expanded the screening of possible hypertrophic mediators to include $\mathrm{CnA}$ expression, the catalytic subunit of $\mathrm{CaN}$ (Supplemental Figure 9D), and the expression and phosphorylation levels of the signaling molecule extracellular signal-regulated kinases 1/2 (ERK1/2) (Supplemental Figure 9, E and F) and protein kinase B (Akt) (Supplemental Figure 9, G and H). Again, there were no significant differences among genotypes. We therefore moved upstream in the signaling cascade and measured the expression of CaM. Remarkably, CaM was increased in hearts from mice over 1 year of age but not in mice younger than 3-6 months (Figure 6, A and C). This difference was only significant in Homo mice, consistent with the more prominent phenotype discussed in the previous section. Transgenic overexpression of $\mathrm{CaM}(27,28)$ and increased levels of free CaM dissociated from RyR2 channels (29) have been linked to cardiac hypertrophy due to activation of downstream signaling. Other hypertrophic signaling pathways reported in a mouse model of severe cardiac hypertrophy due to inability of RyR2 to bind CaM (30) were not altered in P1124L mice (Supplemental Figure 9, C and D).

Cellular $\mathrm{Ca}^{2+}$ handling in ventricular myocytes. The cytosolic loss-of-function phenotype displayed by P1124L channels could lead to reduced $\mathrm{Ca}^{2+}$ release, contractility, and compensatory hypertrophy. Hence, 
A

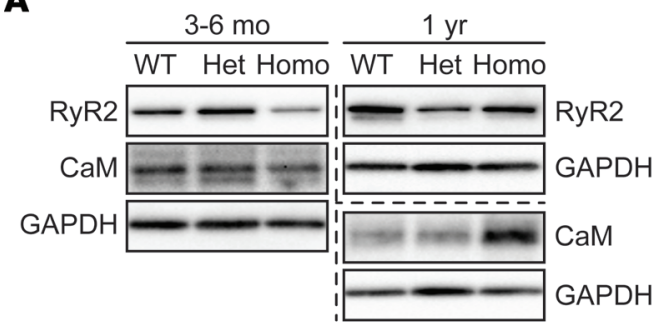

B

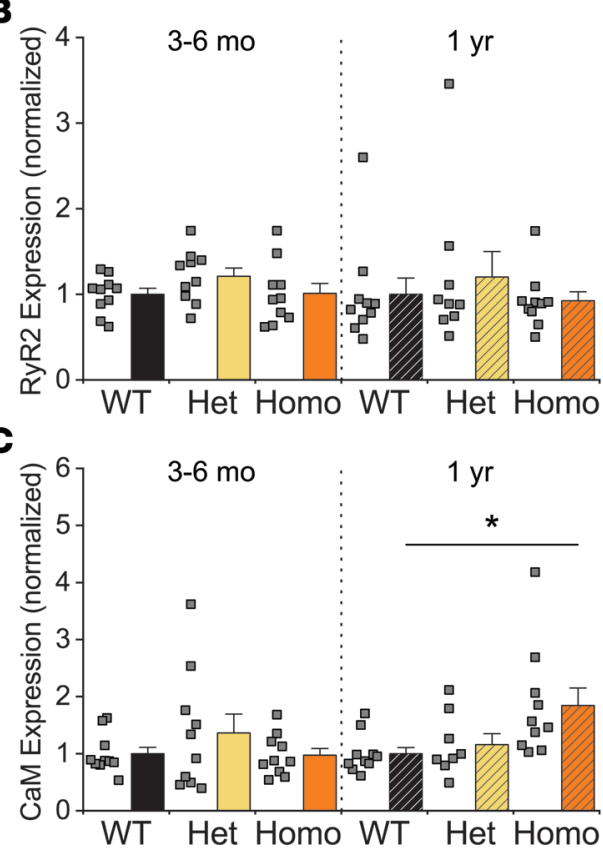

Figure 6. Expression of RyR2 and calmodulin in P1124L hearts. (A) Representative Western blots of ryanodine receptor 2 (RyR2) and calmodulin (CaM) expression in heart homogenates from mice under at 3-6 months of age and over 1 year of age. Band intensities were normalized to the GAPDH signal from the same gel. A separate loading control (CAPDH) is shown for proteins run in different gels. (B) Quantification of RyR2 expression (1-way ANOVA [3-6 months] or ANOVA on ranks [1 year]). (C) Quantification of CaM expression ( $n=10$ WT, 8 [3-6 months] and 9 [1 year] Het, 10 Homo hearts for each age group. ${ }^{*} P<0.05$, ANOVA on ranks).

we measured the kinetics of $\mathrm{Ca}^{2+}$ handling in isolated myocytes from 1-year-old mice to determine whether P1124L induces alterations of intracellular $\mathrm{Ca}^{2+}$ homeostasis. We first paced the cells at $1 \mathrm{~Hz}$ to quantify the properties of the cellular $\mathrm{Ca}^{2+}$ transient at basal conditions and in the presence of isoproterenol (Iso). We observed no significant differences in $\mathrm{Ca}^{2+}$ release, $\mathrm{Ca}^{2+}$ removal, or SR content between WT and P1124L myocytes (Supplemental Figure 10 and Supplemental Table 4). Next, we studied the propensity for SCR during a 30-second resting period, following a train of $3 \mathrm{~Hz}$ stimulation (Figure 7A). The propensity for SCR was higher in Homo myocytes at basal conditions, compared with WT and Het (Figure 7B). This difference was reduced by Iso; however, the frequency of SCR was still significantly higher in Homo myocytes treated with Iso than in WT (Figure 7C). Het myocytes displayed an intermediate phenotype, with a nonsignificant tendency for higher SCR frequency under Iso compared with WT. Interestingly, SCR frequency was not statistically different between Het and Homo under Iso.

Arrhythmia susceptibility of P1124L mice. The high incidence of diastolic $\mathrm{Ca}^{2+}$ waves in RyR2-P1124L cardiomyocytes suggests that mice harboring the mutation may be more susceptible to develop cardiac arrhythmia. We used a challenge protocol (i.p. injection of epinephrine $2 \mathrm{mg} / \mathrm{kg}$ and caffeine $120 \mathrm{mg} / \mathrm{kg}$ ) (31) during surface ECG recording to determine the susceptibility of P1124L mice to develop ventricular arrhythmia. We performed this test in 8-month-old mice because, at this age, they do not show signs of cardiac hypertrophy (Supplemental Table 2). Figure 8A shows representative 2-second lead-II ECG traces. Most of the arrhythmia episodes in P1124L mice involved ventricular bigeminy (alternated premature and sinus beats) and bidirectional 
A
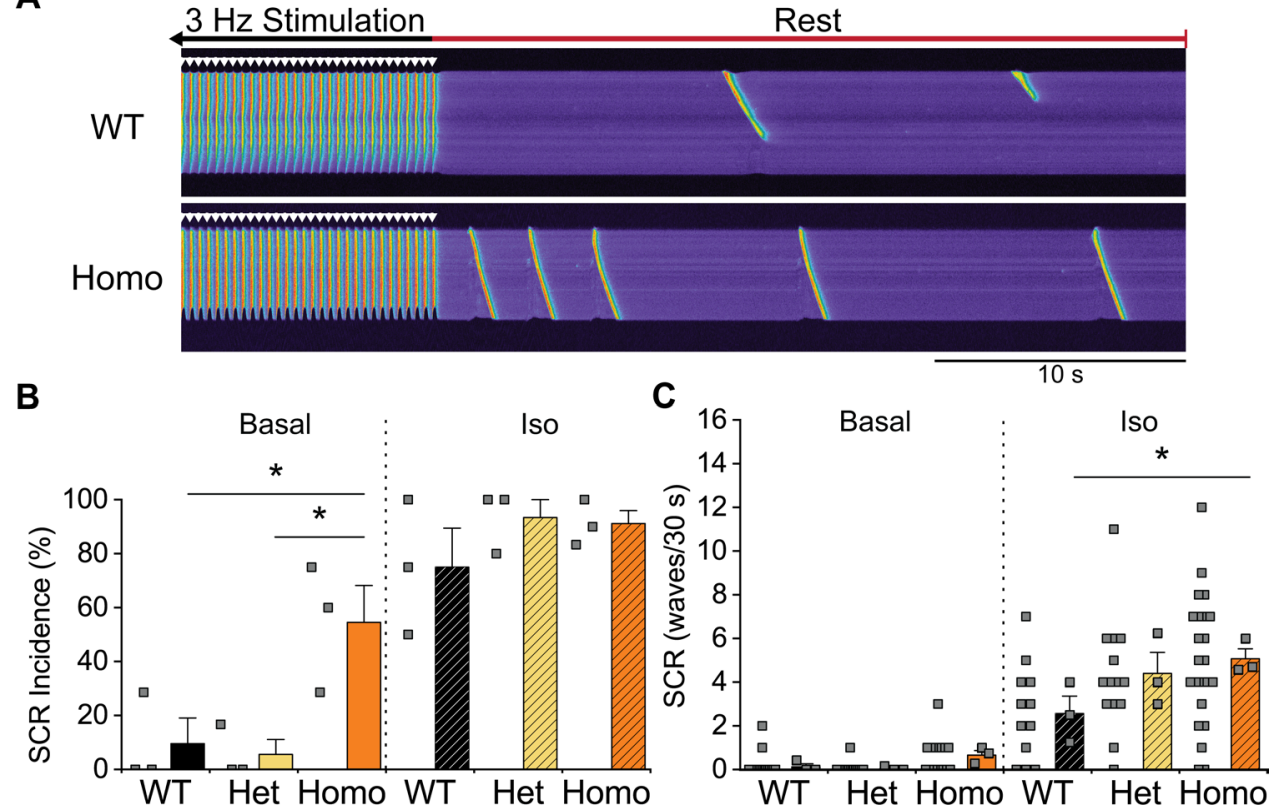

Figure 7. Susceptibility to spontaneous $\mathrm{Ca}^{2+}$ release in ventricular myocytes from 1-year-old mice. (A) Representative traces of WT and Homo ventricular myocytes stimulated with $300 \mathrm{nM}$ isoproterenol (Iso), paced at $3 \mathrm{~Hz}$ for 30 seconds, and monitored at rest for 30 seconds. Enzymatically isolated cardiac myocytes were loaded with the fluorescent $\mathrm{Ca}^{2+}$ indicator fluo-4 AM and field stimulated as indicated. Line-scan images were recorded using a confocal microscope. (B) Percentage of cells showing spontaneous $\mathrm{Ca}^{2+}$ release events (SCR, defined as $\mathrm{Ca}^{2+}$ waves) during the monitoring period. SCR incidence is increased in Homo mice in basal conditions $\left(n=3\right.$ hearts per genotype. ${ }^{*} P<0.05$, 1-way ANOVA). (C) Average number of SCR events in 30 seconds of monitoring after a $3 \mathrm{~Hz}$ train of stimulation (left, individual measurements from $n=17$ WT, 16 Het, 16 Homo basal cells and 12 WT, 14 Het, 23 Homo iso-stimulated cells; right, mean values for $n=3$ hearts per genotype. ${ }^{*} P<0.05$, 1-way ANOVA).

ventricular tachycardia (BDVT). The effect of the treatment was minimal in WT mice, which only showed sporadic premature ventricular beats (PVBs; Figure 8, A and B) and nonsustained arrhythmias (Figure 8C). P1124L mice exhibited a nonsignificant tendency to develop more PVBs than WT (Figure 8B), which coalesced into significantly more episodes of arrhythmia in Homo mice, including BDVT (Figure 8, C-E), the pathognomonic sign of RyR2-induced arrhythmia. Again, Het mice had an intermediate phenotype between WT and Homo. While the difference in the number of episodes of sustained arrhythmia and incidence of BDVT was not statistically different between WT and Het mice, WT animals did not show these types of events, consistent with the literature (Figure 8, D and E). This suggests that the phenotype of Het mice is noteworthy.

In summary, RyR2-P1124L predisposes 8-month-old mice to develop cardiac arrhythmia before the onset of hypertrophy at 1 year of age. This suggests that the arrhythmia is a result of RyR2 dysfunction rather than cardiac structural remodeling. Furthermore, these data suggest that the cellular mechanism for the ventricular arrhythmias in P1124L mice is diastolic SCR.

\section{Discussion}

In 2006, Fujino et al. reported a RyR2 mutation in a family with HCM, T1107M (13). Two subsequent, separate studies showed that this mutation alters the structure of the SPRY2 domain (19) and produces a loss-of-function phenotype (14). Medeiros-Domingo et al. later identified this mutation in patients with CPVT, the inherited arrhythmia already strongly associated with RyR2 dysfunction (16), weakening its exclusive association with HCM. T1107M is not a conserved residue among species, and 2 studies questioned its pathogenicity altogether because it appears with relatively high frequency in WES cohorts (seen in 118 of 277,072 alleles; frequency of 0.0004$)(15,32,33)$, higher than the prevalence of CPVT. In this report, we thoroughly characterized the potentially novel RyR2-P1124L mutation, detected in a patient with obstructive HCM. P1124L also appears in publicly available exome databases, although with a $\sim 20$-fold-lower frequency than T1107M, and we propose its association with a disease with much higher prevalence than CPVT. Hence, to our knowledge, this is the first comprehensive study of a RyR2 
A
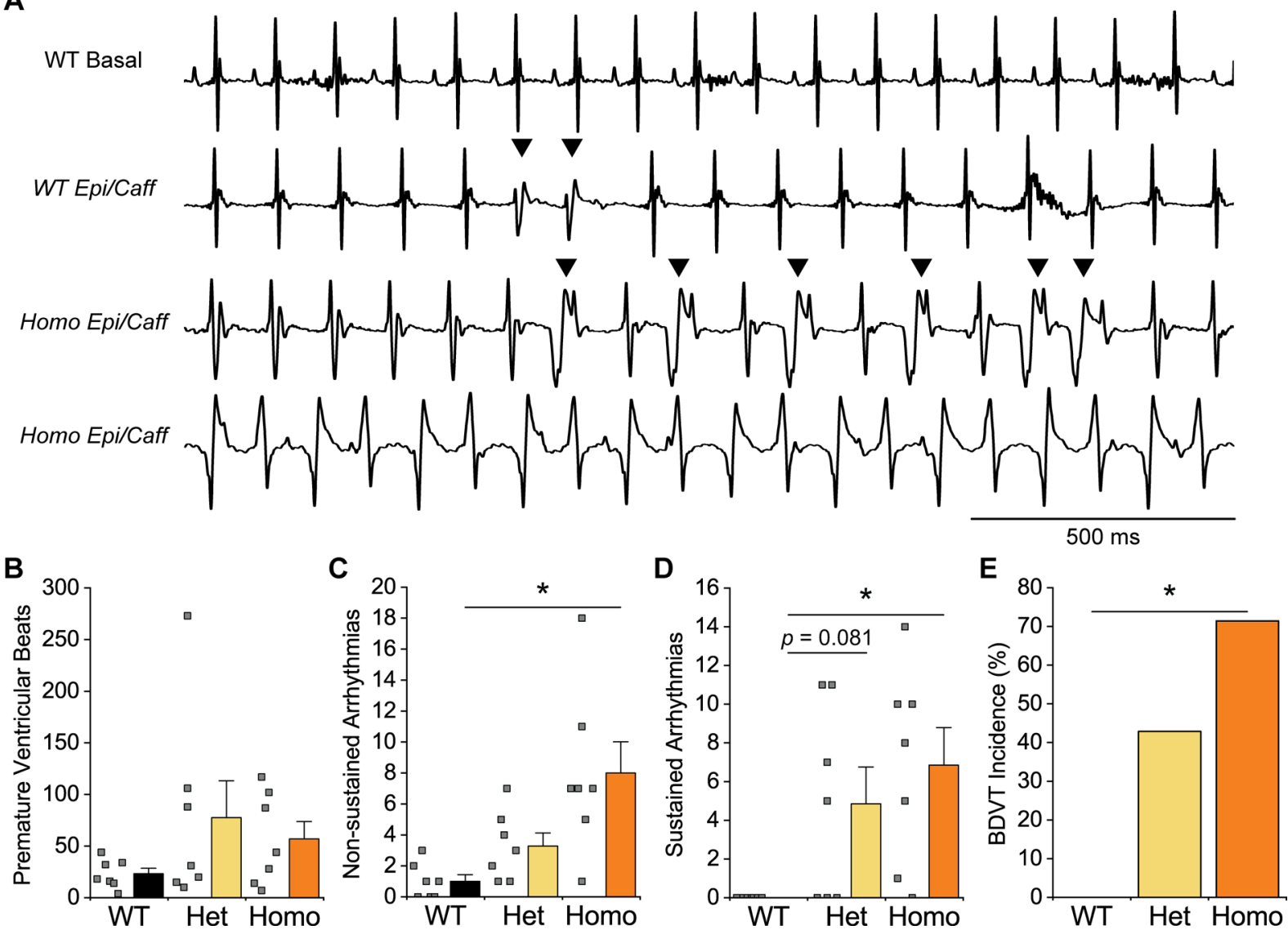

Figure 8. Susceptibility to ventricular arrhythmia in P1124L mice challenged with epinephrine/caffeine at 8 months of age. (A) Two-second representative lead-II ECG traces from mice undergoing arrhythmia challenge. Mice were anesthetized using isoflurane, and the basal ECG was monitored for 10-15 minutes. The ECG was further monitored for 30 minutes, following an i.p. injection of epinephrine (2 mg/kg) and caffeine (120 mg/kg) (Epi/ Caff). From top to bottom, the traces indicate sinus rhythm; premature ventricular beats; nonsustained ventricular bigeminy; and bidirectional ventricular tachycardia (BDVT). (B-D) Average number of premature ventricular beats and episodes of nonsustained (lasting $\leq 5$ seconds) and sustained arrhythmias (lasting $>5$ seconds) in mice undergoing arrhythmia challenge. (E) Incidence of sustained BDVT, defined as ectopic beats with alternating axes of depolarization ( $n=7$ mice per genotype. ${ }^{*} P<0.05$ vs. WT, ANOVA on ranks [B-C], 1-way ANOVA [D], z test [E]).

mutation strongly associated with HCM, a structural disorder of the heart. We provide multiple layers of evidence, from single molecule to whole animal, suggesting a potential association between P1124L and HCM. Additionally, our data show that P1124L underlies an arrhythmogenic phenotype, suggesting that some RyR2 mutations may lead to presentations that cross the boundaries from purely functional (CPVT) to structural (HCM) alterations.

First, we show that P1124L involves a highly conserved residue among RyR isoforms and species and is located within SPRY2, one of the peripheral domains of RyR2, where it produces structural disturbances in the arrangement of a flexible linker at the SPRY2-SPRY3 interface. P1124L is one of a handful of mutations that fall outside of the canonical CPVT1-associated mutation hotspots of RyR2 (Figure 1A), which contain $\sim 93 \%$ of the disease-causing mutations. Our model of the SPRY2-SPRY3 interface suggests that P1124L promotes intradomain rearrangement and alters interdomain interactions that could affect allosteric coupling mechanisms in the channel. The large size of RyR2 makes it difficult to model single amino acid mutations using the whole channel protein; hence, the long-range effects of P1124L are not known. Even then, it is conceivable that the peripheral domains of RyR2 allosterically regulate channel gating or its association with protein partners. This idea is supported by the model suggesting that phosphorylation of S2814, also located in a peripheral domain of RyR2, may turn the channel "leaky" in some forms of heart disease (34). Ultimately, the consequence of the structural alterations to the SPRY region produced by P1124L appears to be a cytosolic loss-of-function, as we determined using 2 complementary assays, $\left[{ }^{3} \mathrm{H}\right]$ ryanodine binding with recombinant protein and single channel recordings with mouse SR microsomes, 
and a SR luminal gain-of-function, which we measured in the SCR assay in HEK293 cells. This is a rather distinct phenotype that, to our knowledge, has only been identified in 1 other mutation of variable penetrance linked to CPVT1 $(24,35)$. While our experiments were carried out using Homo P1124L channels, in the Het condition, as in the patient, any phenotype will be the aggregate result of the absolute expression of each allele and the resulting heterogeneous population of channels containing none to 4 mutant subunits. We have previously addressed this issue for 2 other CPVT1-related RyR2 mutations $(31,36)$.

Second, we show that mice expressing RyR2-P1124L channels develop cardiac hypertrophy at 1 year of age. This phenotype, albeit mild, involves a significant increase in LV wall thickness, myocyte size, and atrial natriuretic peptide (ANP) expression in Homo mice. Het hearts (resembling the genotype of the human patient), on the other hand, show only a tendency for hypertrophy. As there are significant differences between mouse and human cardiac physiology that can modify the effect of specific mutations, it is not entirely surprising that mice may show a milder phenotypic penetrance. For example, mutations that truncate MyBP-C are overtly hypertrophic in Het humans (37) but produce cardiomyopathy only in Homo mice (38-40). Additionally, the penetrance and expressivity of a specific mutation may be affected by the genetic background of the mouse model, as has been previously recognized (41). Indeed, we have observed phenotypic differences between P1124L in the C57B1/6J genetic background used in this study and a strain with a mixed Sv129/C57Bl/6J background (FJA, unpublished observations).

To our knowledge, this report is the first instance in which a clinically relevant RyR2 mutation produces cardiac hypertrophy of any degree in an animal model. In our search for the possible signaling pathways implicated in the hypertrophic phenotype shown by $\mathrm{P} 1124 \mathrm{~L}$ mice, we found that CaM expression is increased in Homo mice at 1 year of age. CaM is a ubiquitously expressed signaling molecule with multiple partners in cardiac cells. Among its many functions, CaM works as the $\mathrm{Ca}^{2+}$ sensor of CaMKII and CaN, 2 signaling molecules implicated in hypertrophic signaling (26). Indeed, both overexpression of CaM $(27,28)$ and its dissociation from membrane-bound partners (29) are linked with cardiac hypertrophy. Our data suggest that an increase in $\mathrm{CaM}$ expression may be implicated in the development of hypertrophy in $\mathrm{P} 1124 \mathrm{~L}$ mice. A hypertrophic phenotype could occur compensatory to a lower cellular/cardiac contractility, resulting from a decreased systolic $\mathrm{Ca}^{2+}$ release. This could occur in the P1124L mouse model as a result of the cytosolic loss-of-function we uncovered at the molecular level. However, the $\mathrm{Ca}^{2+}$ transient data (Supplemental Figure 1 and Supplemental Table 4) suggest that there are no differences in the force of contraction, provided there are no changes in the sensitivity of the myofilaments to $\mathrm{Ca}^{2+}$. At this point, we have no evidence of such alterations. The link between the unique biophysical properties uncovered for P1124L, the increase in CaM expression, and the hypertrophic phenotype observed in P1124L remains to be elucidated. We surmise that subjecting P1124L mice to chronic stress through drug treatments or a surgical approach to induce heart failure may provide additional insight into the pathogenicity of the mutation. Together with the possible influence of the genetic background over the phenotype of the mouse model, as discussed previously, this notion will require further studies.

Finally, we observed cardiac arrhythmia, particularly BDVT, in 8-month-old P1124L mice undergoing arrhythmia challenge. In HCM, ventricular arrhythmias often develop because of the extensive structural and electrophysiological remodeling (42). Interestingly, P1124L mice show both functional and structural phenotypes independently (arrhythmia at 8 months and hypertrophy at 1 year, respectively). Unlike hypertrophy, it is not unexpected to link RyR2 mutations with cardiac arrhythmias since many have been documented to cause CPVT1. From the molecular phenotypes we uncovered for P1124L channels, cytosolic loss-of-function and luminal gain-of-function, the latter may be more prone to drive the arrhythmia process; spontaneous activation of RyR2-P1124L during diastole may produce larger $\mathrm{Ca}^{2+}$ releases that can diffuse to neighboring RyR2 clusters and propagate as arrhythmogenic $\mathrm{Ca}^{2+}$ waves. These events may activate NCX during diastole and produce triggered activity due to delayed afterdepolarizations. Indeed, we observed a high incidence of diastolic SCR in myocytes from P1124L hearts even under basal conditions; however, it was Iso treatment that uncovered an evident increase in the frequency of such events. These observations are consistent with the mechanisms broadly assigned to gain-offunction RyR2 mutations (10). In contrast, mutations that depress channel function favor a mechanism involving sustained bursts of $\mathrm{Ca}^{2+}$ leak after the peak of a $\mathrm{Ca}^{2+}$ transient, leading to early afterdepolarizations (36). In both cases, however, a form of stress (e.g., arrhythmia challenge or Iso) seems necessary to create the substrate required to promote aberrant $\mathrm{Ca}^{2+}$ release and arrhythmia.

As expected from the literature, WT mice did not show BDVT. However, both Het and Homo mice did show this type of arrhythmia, although the incidence was not statistically significant between Het and 
WT mice. In Het mice, permutation analysis predicts that most RyR2 channels should contain at least 1 P1124L subunit and $\sim 6 \%$ of the channels should contain 4 P1124L subunits $(31,36)$. This distribution may be skewed if 1 allele is expressed more predominantly; nonetheless, in the cellular mechanism discussed above, Homo channels are likely driving the arrhythmogenic phenotype.

Clinical relevance. Following its discovery in a patient with HCM, P1124L was classified as a VUS based on ACMG guidelines (17). Our characterization of P1124L in different model systems adds an important layer of evidence to classify P1124L as likely pathogenic (III) in the context of HCM, with one strong (PS3; well-established in vitro and in vivo studies supportive of a damaging effect) and 2 supporting (PP3, PP4) pathogenic criteria. The presence of both hypertrophy and increased susceptibility to arrhythmia in P1124L mice suggest that certain RyR2 variants could lead to both phenotypes, as discussed next. This is a tantalizing idea that requires further research, but the significant differences in mouse and human cardiac physiology and $\mathrm{Ca}^{2+}$ handling could make the mouse a less-than-ideal model to address these questions.

Since the identification of the first mutation associated with HCM affecting myosin heavy chain 7 in 1990 (MYH7-R403Q) (43), the number of genes and variants associated with HCM continues to grow. Even as HCM is overwhelmingly recognized as a monogenic disease caused primarily by mutations in sarcomeric genes, the fact that nearly half of the patients lack a genetic diagnosis cannot be overlooked. This large proportion of mutation-negative cases suggests that HCM has unknown genetic causes if we continue to consider HCM predominantly a monogenic disease; alternatively, it suggests that HCM may also appear as a complex disorder in which genetic modifiers and factors such as age, sex, comorbidities, and lifestyle intervene in the phenotypic outcome (44). This idea is increasingly recognized, and the variable inheritance of nonsarcomeric HCM may favor a multifactorial model $(42,45)$.

As we propose to consider a potentially novel association between RyR2 mutations and nonsarcomeric HCM, and due to the mild hypertrophic phenotype present only in Homo mice, it is important to consider the possibilities outlined above. The role of RYR2 as a CPVT gene is robust; therefore, the increased susceptibility of P1124L mice to arrhythmia is not surprising. However, some mutations, such as P1124L, might in fact present not only as proarrhythmic variants, but also as HCM modifiers. In this scenario, $\mathrm{P} 1124 \mathrm{~L}$ may generate a prohypertrophic substrate in association with other variables, fitting within a multifactorial HCM model. Accordingly, the rare finding of P1124L in WES cohorts with variant-positive subjects displaying a subclinical phenotype or no phenotype at all could be explained by the absence of those yet-to-be-determined factors. Variable phenotypic penetrance is already recognized, but it is incompletely understood, even for well-established HCM genes; previous studies have described families carrying MyBP-C variants where some individuals show the characteristic early onset of hypertrophy, while others remain asymptomatic even as adults (46-48). Multiple studies have shown that, even among mutations in known HCM-associated genes, patients with $>1$ variant generally have a more severe phenotype compared with those with a single identified variant $(49,50)$. We speculate that this type of variation may occur in the patient's family, since the positive family history of HCM on the maternal side suggest that the mother may be a carrier of the mutation with decreased or subclinical disease expressivity. Further screening of Het individuals carrying P1124L may show a similar phenomenon and perhaps a mixed HCM/CPVT phenotype, as seen in the P1124L mouse model. Unfortunately, an expanded genotypic and phenotypic familial assessment was not possible in this study, and we cannot test the possibility that the patient acquired a de novo mutation or shows genetic mosaicism.

In conclusion, although our data do not provide unequivocal evidence to classify $R Y R 2$ as an HCM-causing gene, they present a compelling argument for the importance of studying and understanding the possible implication of rare genes in different forms of HCM. Ultimately, this is an important effort to elucidate the variable disease penetrance and determine the causes of genotype-negative HCM.

\section{Methods}

Identification of RyR2-P1124L in a human patient with clinically diagnosed HCM. Following previous studies, 36 patients (systematically evaluated in Mayo Clinic's HCM Clinic) with clinically diagnosed HCM and a clinical HCM phenotype score predicting a $\geq 60 \%$ probability of having a positive HCM genetic test remained genotype negative for the established HCM-susceptibility genes (7). WES and variant filtering at Mayo Clinic was performed on this cohort of genotype negative/phenotype positive HCM. Following identification of the RyR2-P1124L variant in 1 of the patients, variant adjudication regarding its potential 
pathogenicity followed the 2015 guidelines on variant interpretation of the American College of Medical Genetics and Genomics (ACMG; ref. 17).

Reagents. All chemicals were obtained from MilliporeSigma, unless specified otherwise. Cell culture reagents (media, sera, and antibiotics) were purchased from Thermo Fisher Scientific.

Crystallization of SPRY2. Mouse RyR2 SPRY2 domain (residues 1080-1253) including the P1124L substitution was cloned, expressed, and purified according to a similar strategy previously used for the WT RyR2 SPRY2 domain (19). Protein crystals were grown using the hanging drop method at $20^{\circ} \mathrm{C}$. P1124L (5 $\mathrm{mg} / \mathrm{ml}$ ) was crystallized in $0.1 \mathrm{M}$ potassium thiocyanate and $25 \%-35 \%$ PEG $2000 \mathrm{MME}$ with seeding of WT crystals. Crystals were harvested and flash frozen in the original growth condition supplemented with 25\%-30\% glycerol. Diffraction data were collected at the Advanced Photon Source (APS) beamline 23IDD. Data were processed with HKL2000. The WT RyR2 SPRY2 structure (PDB ID 4P9I; https://www. rcsb.org/structure/4P9I) was used as a search model for molecular replacement to solve the RyR2-SPRY2P1124L mutant structure. The structure was refined using successive rounds of manual building in COOT (51) and automated refinement using Phenix (52). The statistics for data collection and refinement is shown in Supplemental Table 1. Coordinate and structure factor for P1124L is available in the Protein Data Bank (www.rcsb.org) with accession code 5VSN.

Transient expression of RyR2 in HEK293 cells. HEK293 cells were grown in DMEM supplemented with $10 \% \mathrm{FBS}, 100 \mathrm{U} / \mathrm{ml}$ penicillin, and $100 \mu \mathrm{g} / \mathrm{ml}$ streptomycin on $100-\mathrm{mm}$ tissue culture dishes. Cells were transfected with a plasmid containing the mouse Ryr2 cDNA using the Xtreme-DNA reagent (Roche Diagnostics), following the instructions of the manufacturer. Cell lysates were prepared from transfected cells, as previously described (36). Forty-eight hours after transfection, cells were washed twice with PBS and then scraped from the plate and solubilized in $200 \mu \mathrm{l}$ of lysis buffer, containing $25 \mathrm{mM}$ Tris/50 mM HEPES (pH 7.4), $137 \mathrm{mM} \mathrm{NaCl}, 1 \%$ CHAPS, 0.5\% soybean phosphatidylcholine, $2.5 \mathrm{mM}$ DTT, and protease inhibitors $(2 \mu \mathrm{M}$ leupeptin, $100 \mu \mathrm{M}$ phenylmethylsulphonyl fluoride, $500 \mu \mathrm{M}$ benzamidine, and $100 \mathrm{nM}$ aprotinin). The cells were incubated on ice for 1 hour, and lysates were obtained by centrifuging at $16,000 \mathrm{~g}$ at $4^{\circ} \mathrm{C}$ for 10 minutes to remove insoluble material. Protein concentrations were determined using the Bradford method (Bio-Rad).

$\left[{ }^{3} H\right]$ Ryanodine binding assays. Binding assays were carried out following a modified version of a protocol previously described (53). Binding mixtures were prepared containing 50-100 $\mu$ g of cell lysate, 0.2 M KCl, $20 \mathrm{mM}$ Na-HEPES (pH 7.4), $6.5 \mathrm{nM}\left[{ }^{3} \mathrm{H}\right]$ ryanodine (NET950, PerkinElmer), and enough $\mathrm{CaCl}_{2}$ to set free $\left[\mathrm{Ca}^{2+}\right]$ between $100 \mathrm{nM}-100 \mu \mathrm{M}$. EGTA $(1 \mathrm{mM})$ was used to buffer $\mathrm{Ca}^{2+}$. The $\mathrm{Ca}^{2+} /$ EGTA ratio for these solutions was determined using MaxChelator (WEBMAXCLITE v1.15, https:// somapp.ucdmc.ucdavis.edu/pharmacology/bers/maxchelator/webmaxc/webmaxclite115.htm). The binding reactions were incubated for 2 hours at $37^{\circ} \mathrm{C}$ and were then filtered through Whatman GF/B filters presoaked in $5 \%$ polyethyleneimine to maximize protein retention and washed 3 times with $5 \mathrm{ml}$ of distilled water in a Brandel M24-R Harvester. Nonspecific binding was determined in the presence of $20 \mu \mathrm{M}$ unlabeled ryanodine (2153770, MP Biomedicals). $\left[{ }^{3} \mathrm{H}\right]$ ryanodine binding was measured by liquid scintillation using Bio-Safe II counting cocktail (RPI Research). Corrections for RyR2 expression were determined by dividing the total $\left[{ }^{3} \mathrm{H}\right]$ ryanodine binding by the intensity from Western blots, expressed as a percentage of the WT. Hill's equation was used to determine the maximum $\left[{ }^{3} \mathrm{H}\right] \mathrm{ryano}$ dine binding and the $\mathrm{EC}_{50}$ in Origin $2018 \mathrm{~b}$ (Origin Lab).

SCR in HEK293 Cells. Stable inducible HEK293 cells lines expressing the WT (53) or P1124LRyR2 were cultured as described above. SCR was measured using the ER-targeted FRET Ca ${ }^{2+}$ sensor D1ER $(25,54)$. After 18-24 hours of subculture on 35-mm glass-bottom dishes, pcDNA-D1ER (36325, Addgene) was transfected as described in the previous section. Eighteen to 24 hours after transfection, RyR2 expression was induced by adding $1 \mu \mathrm{g} / \mathrm{ml}$ tetracycline to the culture medium. For imaging (18-24 hours after induction), cells were continually perfused with Krebs-Ringer-HEPES buffer containing (in mM): $125 \mathrm{NaCl}, 5 \mathrm{KCl}, 1.2 \mathrm{KH}_{2} \mathrm{PO}_{4}, 1.2 \mathrm{MgCl}_{2}, 6$ glucose, and $25 \mathrm{HEPES}$ (pH 7.4). $\mathrm{CaCl}_{2}$ (1 mM and $\left.2 \mathrm{mM}\right), 1 \mathrm{mM}$ tetracaine, and/or $20 \mathrm{mM}$ caffeine were added as indicated. Images were captured at room temperature using a LSM800 confocal microscope (Carl Zeiss) with a Plan-Apochromat 20×/0.8 M27 objective at $405 \mathrm{~nm}$ excitation and with 400-500 nm (cyan fluorescent protein; CFP) and 500-700 (yellow fluorescent protein; YFP) emission. Time-lapse images were collected every 1.5 seconds with a scanning time of $1.29 \mathrm{~s} /$ frame. FRET was calculated as the YFP/CFP ratio. The maximum store capacity, fractional release, and activation and termination thresholds were 
calculated using the following equations, where $\mathrm{F}_{\text {Act }}$ and $\mathrm{F}_{\text {Term }}$ are the FRET levels when spontaneous $\mathrm{Ca}^{2+}$ oscillations activate and terminate, $\mathrm{F}_{\mathrm{Max}}$ is the maximum FRET level with tetracaine perfusion, and $\mathrm{F}_{\mathrm{Min}}$ is the residual FRET level after the ER is emptied with caffeine.

$$
\begin{aligned}
& \text { Store Capacity }=\left(F_{\text {Max }}-F_{\text {Min }}\right) \quad \text { Termination Threshold }=\frac{F_{\text {Term }}-F_{\text {Min }}}{\text { Store Capacity }} \times 100 \% \\
& \text { Activation Threshold }=\frac{F_{\text {Act }}-F_{\text {Min }}}{\text { Store Capacity }} \times 100 \% \quad \text { Fractional Release }=\frac{F_{\text {Act }}-F_{\text {Term }}}{\text { Store Capacity }} \times 100 \%
\end{aligned}
$$

Generation of the RyR2-P1124L targeted mice. The RyR2-P1124L-KI targeting vector was constructed using a combination of traditional cloning techniques and recombineering, a highly efficient phage-based Escherichia coli homologous recombination system $(55,56)$. The RyR2-P1124L-KI targeting vector was linearized with NotI and electroporated into murine SV/129 AB2.2 embryonic stem (ES) cells (57). ES cells that integrated the targeting vector either by homologous or random integration were selected by growth on G418. Gancyclovir (GANC) selected against clones that contained the HSV-TK cassette, thus enriching for clones that integrated the Neo cassette by homologous recombination. Four hundred seventy-two neomycin resistant $\left(\mathrm{Neo}^{\mathrm{r}}\right)$, gancyclovir resistant $\left(\mathrm{GANC}^{\mathrm{r}}\right)$ colonies were selected, replicated and expanded. DNA was isolated from 192 replica ES clones, digested with BamHI, electrophoresed on agarose gels, transferred to charged nylon membranes, and hybridized to radiolabeled 3' probe. Thirty-six correctly targeted clones were identified by a 17.2$\mathrm{kb}$ band, in addition to the $21.7-\mathrm{kb}$ native band. DNA from 19 clones that appeared to be correctly targeted on the $3^{\prime}$ side were digested with $\mathrm{Nco} 1$ electrophoresed on agarose gels, transferred to charged nylon membranes, and hybridized to radiolabeled $5^{\prime}$ probe. Nineteen positive clones were identified. Six clones (2B2, $2 \mathrm{C} 3,2 \mathrm{E} 5,2 \mathrm{~B} 9,2 \mathrm{C} 9$, and $2 \mathrm{G} 9 \mathrm{FF}$ ) were thawed from the master plate and fully expanded. The remaining 13 clones were partially expanded and cryopreserved. The expanded clones were genotyped using both the 5' and $3^{\prime}$ probes. Correctly targeted clones were identified by the presence of a $21.7-\mathrm{kb}$ native band and a 17.2$\mathrm{kb}$ altered band when digested with BamH1; they were then transferred to a charged nylon membrane and hybridized to the $3^{\prime}$ probe in the presence of a $15.2-\mathrm{kb}$ native band and an $8.7-\mathrm{kb}$ altered band when digested with $N c 01$ and hybridized with the 5' probe. DNA sequence analysis was used to identify the correctly targeted clones and to confirm the presence of the floxed Neo cassette and the P to L substitution at position 1124 . The chromosomes of 2 Ryr2-P1124L clones were counted, and both clones were found to be euploid. Clones RyR2-P1124L 2E5 and RyR2-P1124L 2B2 were microinjected into C57B1/6J blastocyst to produce chimeric founders. Highly chimeric males were crossed with C57B1/6J females (000664, the Jackson Laboratory). Agouti pups carrying the P1124L were identified by PCR. The floxed Neo cassette was excised by mating Het RyR2-P1124L mice with EIIa-Cre transgenic mice (003724, the Jackson Laboratory). Pups with complete Neo excision were identified by PCR and then backcrossed with C57B1/6J mice. RyR2-P1124L pups negative for the Cre recombinase gene were further backcrossed for 7 additional generations with C57Bl/6J mice to obtain a $99 \%+$ congenic strain (58). To confirm the presence of $\mathrm{P} 1124 \mathrm{~L}$, the region of Ryr2 containing the mutation was amplified with PCR and sequenced using the following primers: 5'-CGCATCTTCAGGGCAGAG-3' and 5'-GAGTGAAGCGGGAATGTC-3'. The sequences obtained were compared with the targeting vector, Ryr2 (chromosome 13, accession number NT_039578.8), as well as the corresponding mRNA (accession number NM_023868.2) using Nucleotide BLAST (http://blast.ncbi.nlm.nih.gov/Blast.cgi). The translated protein was compared with the mouse RyR2 (accession number E9Q401).

Mouse phenotyping. Transthoracic echocardiography (Echo) was performed by the Echocardiography Service of the Frankel Cardiovascular Center Animal Phenotyping Core at the University of Michigan using a Vevo 2100 system with a 22-55 MHz transducer (MS550D, Visual Sonics), as described previously (59). Mice were anesthetized with 1\%-2\% isoflurane and maintained on a heated platform. Two-dimensionally guided M-mode images of the LV were acquired at the tip of the papillary muscles. LV diameter, septum wall, and posterior wall thickness were recorded to determine the fractional shortening, LV volume, $\mathrm{EF}$, and HR. All parameters were measured over at least 3 consecutive cycles.

Surface electrocardiograms were recorded as described previously (31). Mice were anesthetized with isoflurane $(2 \%-5 \%)$ and maintained on a heated pad. Needle electrodes were placed under the skin to record in Lead-I and Lead-II configurations, using a PowerLab system (ADInstruments). After 5-10 minutes of stable baseline recording, epinephrine $(2 \mathrm{mg} / \mathrm{kg})$ and caffeine $(120 \mathrm{mg} / \mathrm{kg})$ were applied i.p. Animals were monitored for 30 minutes after injection in search of arrhythmic events (typically ventricular 
bigeminy and ventricular tachycardia) defined as 3 or more ventricular ectopic beats, consecutive or alternated with normal beats. These events were further classified as nonsustained (lasting less than 5 seconds) or sustained (lasting 5 seconds or longer).

For histological staining, formalin-fixed paraffin-embedded (FFPE) hearts were sectioned, stained, and imaged by the Unit of Laboratory Animal Medicine In-Vivo Animal Core at the University of Michigan. An expert pathologist performed a detailed analysis of the slides and quantified the level of fibrosis.

The experimenter was blinded in all procedures performed at institutional cores. Age- and sex-matched WT littermates and C57B1/6J mice were used as controls. All sets include males and females. Numbers and age of the mice are indicated in each figure.

Tissue homogenization. Whole heart homogenates were prepared as previously described (60). Briefly, frozen hearts were pulverized in liquid nitrogen, suspended in homogenization buffer $(0.9 \% \mathrm{NaCl}$, Tris$\mathrm{HCl} 10 \mathrm{mM}$ [pH 6.8], $20 \mathrm{mM} \mathrm{NaF}, 2 \mu \mathrm{M}$ leupeptin, $100 \mu \mathrm{M}$ phenylmethylsulphonyl fluoride, $500 \mu \mathrm{M}$ benzamidine, $100 \mathrm{nM}$ aprotinin), homogenized using a Teflon pestle, and centrifuged at $1000 \mathrm{~g}$ for 10 minutes at $4^{\circ} \mathrm{C}$. Supernatants were aliquoted and stored at $-80^{\circ} \mathrm{C}$ until used. Protein concentrations were determined using the Bradford method (Bio-Rad).

Western blotting. Tissue homogenate $(50 \mu \mathrm{g})$ was suspended in Laemmli buffer and separated by SDSPAGE in 4-20\% TGX or AnyKD precast gels (Bio-Rad). Proteins were then transferred to PVDF membranes using the iblot2 transfer system (Thermo Fisher Scientific) or overnight wet transfer. Membranes were probed with the following primary antibodies: anti-RyR [clone 34C] (1:2000; MA3-925, Thermo Fisher Scientific), -pS2808-RyR (1:5000; A010-30, Badrilla), -pS2030 (1:1000, custom-made) (60), -pS2814 (1:1000, custom-made) (60), -dpS2030 (1:1000, custom-made) (60), -SERCA2 (clone 2A7-1; 1:1000; ab2861, Abcam), -NCX1 (clone 6H2; 1:200; ab6495, Abcam), -PLB (clone A1; 1:5000; A010-14, Badrilla), -pS16-PLB (1:5000; A010-12, Badrilla), -pT17-PLB (1:5000; A010-13, Badrilla), -Cav1.2 (1:200; ACC-003, Alomone), -CaMKII (clone D11A10; 1:1000; 4436, Cell Signaling Technologies), -pT287-CaMKII (clone D21E4; 1:1000; 12716, Cell Signaling Technologies), -p44/42 MAPK (Erk1/2; 1:1000; 9102, Cell Signaling Technologies), -pT202/ Y204-p44/42 MAPK (Erk1/2; 1:1000; 9101, Cell Signaling Technologies), -Akt (1:1000; 9272, Cell Signaling Technologies), -pS473-Akt (1:1000; 9271, Cell Signaling Technologies), and -GAPDH (clone 6C5; 1:10000; MAB374, MilliporeSigma). Secondary antibodies, used as appropriate, were goat anti-mouse-HRP (1:1000 or 1:5000; 31437, Thermo Fisher Scientific) or goat anti-rabbit-HRP (1:2000 or 1:10000; 31463, Thermo Fisher Scientific). Membranes were developed using SuperSignal Femto ECL reagent (Thermo Fisher Scientific) and imaged with a ChemiDoc MP apparatus (Bio-Rad). Band intensity was quantified with the ImageLab software (Bio-Rad). Custom-made antibodies against the phosphorylated forms of RyR2 are described elsewhere (60).

Single channel recordings. Cardiac SR-enriched microsomes for single channel recordings were prepared from mouse hearts using differential centrifugation, as previously described (36). Ten to 15 pooled mouse hearts were homogenized as described above. Homogenates were centrifuged a second time at $8000 \mathrm{~g}$ for 20 minutes at $4^{\circ} \mathrm{C}$. Supernatants were further centrifuged at $100,000 \mathrm{~g}$ for 35 minutes at $4^{\circ} \mathrm{C}$. The pellets containing SR-enriched microsomes were resuspended in homogenization buffer supplemented with $0.3 \mathrm{M}$ sucrose.

RyR2 channels from SR microsomes were incorporated into planar lipid bilayers to record single channel activity as previously reported (36). The trans $(1000 \mu \mathrm{l})$ and the cis $(1000 \mu \mathrm{l})$ chambers (corresponding to the luminal and cytoplasmic side of the channel) contained 300 and $50 \mathrm{mM} \mathrm{CsCH}_{3} \mathrm{SO}_{3}$, respectively; $20 \mathrm{mM}$ MOPS ( $\mathrm{pH} 7.2)$; and nominally free $\left[\mathrm{Ca}^{2+}\right](\sim 3-5 \mu \mathrm{M})$. A phospholipid bilayer of phosphatidylethanolamine/phosphatidylserine/phosphatadylcholine (1.0:0.8:0.2 ratio dissolved in $\mathrm{n}$-decane to $20 \mathrm{mg} / \mathrm{ml}$ ) was painted with a glass rod across an aperture of $\sim 200 \mu \mathrm{m}$ diameter in a Delrin cup. The trans chamber was the voltage control side connected to the head stage of a 200-A Axopatch amplifier (Molecular Devices), while the cis side was held at virtual ground. Channel activity was recorded after filtration with an 8-pole low-pass Bessel filter set at $1.5 \mathrm{kHz}$ and digitized at a rate of $4 \mathrm{kHz}$ using a Digidata 1200 AD/DA interface (Molecular Devices). Data acquisition and analysis was performed with Axon Instruments hardware and software (pClamp 10, Molecular Devices).

Gene expression analysis. Tissue was homogenized in TRIzol reagent (Thermo Fisher Scientific) following the manufacturer's instructions. After addition of an appropriate amount of chloroform, mixing, incubation, and centrifugation, the RNA-containing aqueous phase was collected and treated with DNase I (Qiagen) for 1 hour. RNA was subsequently purified using the RNeasy mini kit (Qiagen), following the manufacturer's protocol. First-strand cDNA synthesis was performed using the iScript Reverse Transcription Kit (Bio-Rad). To facilitate full-length first-strand cDNA synthesis, samples were incubated for 90 
minutes at $50^{\circ} \mathrm{C}$ prior to heat inactivation of the reverse transcriptase at $70^{\circ} \mathrm{C}$ for 15 minutes. Expression levels of Nppa (Mm01255748_g1, Thermo Fisher Scientific), Nppb (Mm01255770_g1, Thermo Fisher Scientific), Myh7 (Mm00600555_m1, Thermo Fisher Scientific), and Actb (4352933E, Thermo Fisher Scientific) were assessed using TaqMan gene expression probes.

Isolation of ventricular myocytes. Ventricular myocytes were isolated as previously described $(59,61)$. Briefly, mice were heparinized (0.5 U/g i.p., Sagent Pharmaceuticals) and anesthetized with urethane (1 $\mathrm{mg} / \mathrm{kg}$ i.p., MilliporeSigma). The heart was quickly excised, mounted on a Langendorff apparatus, and perfused with Perfusion Buffer (PB) containing (in $\mathrm{mM}$ ) $113 \mathrm{NaCl}, 4.7 \mathrm{KCl}, 1.2 \mathrm{MgSO}_{4}-7 \mathrm{H}_{2} \mathrm{O}, 10 \mathrm{HEPES}$, $0.6 \mathrm{Na}_{2} \mathrm{HPO}_{4}, 12 \mathrm{NaHCO}_{3}, 0.6 \mathrm{KH}_{2} \mathrm{PO}_{4}, 10 \mathrm{KHCO}_{3}, 30$ Taurine, 10 2,3-Butanedione monoxime, and 5.5 glucose ( $\mathrm{pH} 7.46)$ at $37^{\circ} \mathrm{C}$ and $3 \mathrm{ml} / \mathrm{min}$. The heart was then perfused with $\mathrm{PB}$ supplemented with 773.48 $\mathrm{U} / \mathrm{ml}$ Collagenase Type II (Worthington), $0.14 \mathrm{mg} / \mathrm{ml}$ Trypsin (Thermo Fisher Scientific), and $12.5 \mu \mathrm{M}$ $\mathrm{CaCl}_{2}$. Once fully digested (5-7 min), the ventricles were minced in PB buffer containing with $10 \% \mathrm{FBS}$ and $12.5 \mu \mathrm{M} \mathrm{CaCl}_{2}$. Tissue pieces were gently pipetted to dissociate cells. $\mathrm{Ca}^{2+}$ was reintroduced to 1.8 $\mathrm{mM}$ in 5 steps, and cells were kept in Tyrode's solution containing (in $\mathrm{mM}$ ) $135 \mathrm{NaCl}, 4 \mathrm{KCl}, 1.8 \mathrm{CaCl}_{2}, 1$ $\mathrm{MgCl}_{2}, 10 \mathrm{HEPES}, 1.2 \mathrm{NaH}_{2} \mathrm{PO}_{4}$, and 10 glucose ( $\mathrm{pH}$ 7.40) until used.

Confocal $\mathrm{Ca}^{2+}$ imaging. $\mathrm{Ca}^{2+}$ transients triggered by field stimulation were recorded from cardiac myocytes as previously described (59). Cells were incubated in $10 \mu \mathrm{M}$ fluo-4 AM (Thermo Fisher Scientific) with $0.4 \%$ Pluronic F-127 (Thermo Fisher Scientific) at $37^{\circ} \mathrm{C}$ for 5 minutes. Cells were then washed and kept in fresh Tyrode's solution. Longitudinal line-scan images were recorded using a LSM510 Meta confocal microscope (Carl Zeiss) with a $40 \times / 1.2$ water immersion objective at $3.072 \mathrm{~ms} /$ line. Fluorescence was excited at $488 \mathrm{~nm}$ and recorded at $>505 \mathrm{~nm}$. Myocytes were first perfused with Tyrode's solution and paced at $1 \mathrm{~Hz}$ using a Grass Stimulator (2 ms field-stimulation pulses, 60-70 V). When a stable response was established, cells were perfused with Tyrode's solution containing $300 \mathrm{nM}$ Iso for $2 \mathrm{~min}$. Finally, $20 \mathrm{mM}$ caffeine was applied to measure the SR $\mathrm{Ca}^{2+}$ load. The last $5 \mathrm{Ca}^{2+}$ transients under basal conditions and with Iso were averaged for each cell. The fractional release was calculated as a percentage of the SR $\mathrm{Ca}^{2+}$ load released during field stimulation. To determine the susceptibility to develop SCR events, cells were paced at $3 \mathrm{~Hz}$ for 30 seconds at basal conditions and in the presence of $300 \mathrm{nM}$ Iso. Following this train of stimulation, cells were monitored for 30 seconds for $\mathrm{Ca}^{2+}$ waves and triggered activity.

Statistics. Data are presented as mean \pm SEM, with individual data points indicated. Measurements performed in isolated cardiac myocytes are presented as scatter plots, while the average value for each heart is presented over a bar graph showing mean \pm SEM. Data shown in box plots are presented with whiskers indicating the most extreme data points within the 1.5 interquartile range. Statistical significance was determined at $P<0.05$ for comparisons between mice/hearts using $\mathrm{z}$ test, 2-tailed $t$ test, rank sum test, 1-way ANOVA with Holm-Sidak's post hoc, or ANOVA on ranks with Dunn's post hoc. All analyses and assessments of whether data met assumptions were carried out in SigmaPlot 13 (Systat Software). Data were plotted in Origin 2018b (Origin Lab).

Study approval. Human studies were performed under IRB approval number 811-98 from Mayo Clinic. Patients provided a written informed consent prior to the inclusion in this study. Classification of genetic variants was performed following ACMG guidelines (17). Animal use was approved by the University of Michigan IACUC (PRO6075) and the University of Wisconsin-Madison School of Medicine and Public Health IACUC (M5944); both institutions run animal programs accredited by Association for Assessment and Accreditation of Laboratory Animal Care (AAALAC). Animal husbandry was performed by specialized personnel following each institution's standard procedures.

\section{Author contributions}

FJA designed experiments and performed arrhythmia studies, expression, and analysis of recombinant RyR2; confocal imaging; Western blots; and most data analysis and also wrote the manuscript. JMB, CAM, and MJA conducted the human studies and identified the P1124L patient. ZY and FVP crystallized and solved the atomic structure of SPRY2. CRV collected and analyzed the single channel recordings. JJH performed and analyzed the gene expression assays. YTZ participated in the isolation of ventricular myocytes and analysis of confocal images. DSH prepared recombinant protein and maintained cell cultures. YC maintained and genotyped the mouse colony. TRB performed some Western blot measurements. HHV conceived the project and designed experiments, supervised the work, and wrote the manuscript. All authors participated in the editing of the manuscript. 


\section{Acknowledgments}

Funding for this study was provided by the American Heart Association (14PRE19500012 to FJA), the Mayo Clinic Windland Smith Rice Comprehensive Sudden Cardiac Death Program and the Paul and Ruby Tsai and Family Hypertrophic Cardiomyopathy Research Fund (JMB and MJA), and the NIH (HL055438, HL120108, and HL134344 to HHV). We thank Wayne Chen (Department of Physiology and Pharmacology, University of Calgary, Calgary, Alberta, Canada) for providing the RyR2-P1124L plasmid and stable cell lines expressing RyR2; Amy Palmer and Roger Tsien for providing the D1ER plasmid through Addgene (Watertown, Massachusetts, USA); and the Frankel Cardiovascular Center Animal Phenotyping Core and the Unit of Laboratory Animal Medicine In-Vivo Animal Core of the University of Michigan for their services. We also thank the University of Wisconsin-Madison Biotechnology Center Genome Editing and Animal Models Facility for their contributions in generating the P1124L mouse model

Address correspondence to: Francisco J. Alvarado or Héctor H. Valdivia, 1111 Highland Avenue, 8528 WIMR II, Madison, Wisconsin 53705, USA. Phone: 608.262.6924; Email: falvarad@medicine.wisc. edu (FJA), hvaldivia@wisc.edu (HHV). Or to: Michael J. Ackerman, Mayo Clinic, 200 First Street SW, Guggenheim 5-01; Rochester, Minnesota 55901, USA. Phone: 507.284.0101; Email: ackerman.michael@ mayo.edu. Or to: Filip Van Petegem, Life Sciences Office 2356, 2350 Health Sciences Mall, Vancouver, British Columbia V6T 1Z3, Canada. Phone: 604.827.4267; Email: petegem@mail.ubc.ca.

DSH's present address: Department of Pharmacology and Toxicology, Michigan State University, East Lansing, Michigan, USA.

1. Mozaffarian D, et al. Heart disease and stroke statistics--2015 update: a report from the American Heart Association. Circulation. 2015;131(4):e29-322.

2. Ho CY. Hypertrophic cardiomyopathy: preclinical and early phenotype. J Cardiovasc Transl Res. 2009;2(4):462-470.

3. Maron BJ. Sudden death in hypertrophic cardiomyopathy. J Cardiovasc Transl Res. 2009;2(4):368-380.

4. Maron BJ, Maron MS, Semsarian C. Genetics of hypertrophic cardiomyopathy after 20 years: clinical perspectives. $J$ Am Coll Cardiol. 2012;60(8):705-715.

5. Bos JM, Ommen SR, Ackerman MJ. Genetics of hypertrophic cardiomyopathy: one, two, or more diseases? Curr Opin Cardiol. 2007;22(3):193-199

6. Bos JM, Towbin JA, Ackerman MJ. Diagnostic, prognostic, and therapeutic implications of genetic testing for hypertrophic cardiomyopathy. J Am Coll Cardiol. 2009;54(3):201-211.

7. Bos JM, Will ML, Gersh BJ, Kruisselbrink TM, Ommen SR, Ackerman MJ. Characterization of a phenotype-based genetic test prediction score for unrelated patients with hypertrophic cardiomyopathy. Mayo Clin Proc. 2014;89(6):727-737.

8. Landstrom AP, Ackerman MJ. Beyond the cardiac myofilament: hypertrophic cardiomyopathy- associated mutations in genes that encode calcium-handling proteins. Curr Mol Med. 2012;12(5):507-518.

9. Martins AS, et al. In Vivo Analysis of Troponin C Knock-In (A8V) Mice: Evidence that TNNC1 Is a Hypertrophic Cardiomyopathy Susceptibility Gene. Circ Cardiovasc Genet. 2015;8(5):653-664.

10. Alvarado FJ, Valdivia HH. Inheritable Phenotypes Associated With Altered Intracellular Calcium Regulation. In: Zipes DP, Jalife J, Stevenson WG, eds. Cardiac Electrophysiology: from cell to bedside. Philadelphia, PA.: Elsevier; 2018:504-512.

11. Bers DM. Calcium cycling and signaling in cardiac myocytes. Annu Rev Physiol. 2008;70:23-49.

12. Kapplinger JD, et al. Yield of the RYR2 Genetic Test in Suspected Catecholaminergic Polymorphic Ventricular Tachycardia and Implications for Test Interpretation. Circ Genom Precis Med. 2018;11(2):e001424.

13. Fujino N, et al. Abstract 915: A Novel Missense Mutation in Cardiac Ryanodine Receptor Gene as a Possible Cause of Hypertrophic Cardiomyopathy: Evidence From Familial Analysis. Circulation. 2006;114(suppl 18):II_165.

14. Tang Y, Tian X, Wang R, Fill M, Chen SR. Abnormal termination of Ca2+ release is a common defect of RyR2 mutations associated with cardiomyopathies. Circ Res. 2012;110(7):968-977.

15. Landstrom AP, et al. Interpreting Incidentally Identified Variants in Genes Associated With Catecholaminergic Polymorphic Ventricular Tachycardia in a Large Cohort of Clinical Whole-Exome Genetic Test Referrals. Circ Arrhythm Electrophysiol. 2017;10(4):e004742.

16. Medeiros-Domingo A, et al. The RYR2-encoded ryanodine receptor/calcium release channel in patients diagnosed previously with either catecholaminergic polymorphic ventricular tachycardia or genotype negative, exercise-induced long QT syndrome: a comprehensive open reading frame mutational analysis. J Am Coll Cardiol. 2009;54(22):2065-2074.

17. Richards S, et al. Standards and guidelines for the interpretation of sequence variants: a joint consensus recommendation of the American College of Medical Genetics and Genomics and the Association for Molecular Pathology. Genet Med. 2015;17(5):405-424.

18. Yuchi Z, et al. Crystal structures of ryanodine receptor SPRY1 and tandem-repeat domains reveal a critical FKBP12 binding determinant. Nat Commun. 2015;6:7947.

19. Lau K, Van Petegem F. Crystal structures of wild type and disease mutant forms of the ryanodine receptor SPRY2 domain. Nat Commun. 2014;5:5397.

20. Peng W, et al. Structural basis for the gating mechanism of the type 2 ryanodine receptor RyR2. Science. 
2016;354(6310):aah5324.

21. des Georges A, et al. Structural Basis for Gating and Activation of RyR1. Cell. 2016;167(1):145-157.e17.

22. Kimlicka L, Lau K, Tung CC, Van Petegem F. Disease mutations in the ryanodine receptor N-terminal region couple to a mobile intersubunit interface. Nat Commun. 2013;4:1506.

23. Kimlicka L, Tung CC, Carlsson AC, Lobo PA, Yuchi Z, Van Petegem F. The cardiac ryanodine receptor N-terminal region contains an anion binding site that is targeted by disease mutations. Structure. 2013;21(8):1440-1449.

24. Liu Y, et al. CPVT-associated cardiac ryanodine receptor mutation G357S with reduced penetrance impairs Ca2+ release termination and diminishes protein expression. PLoS ONE. 2017;12(9):e0184177.

25. Jones PP, et al. Endoplasmic reticulum Ca2+ measurements reveal that the cardiac ryanodine receptor mutations linked to cardiac arrhythmia and sudden death alter the threshold for store-overload-induced Ca2+ release. Biochem J. 2008;412(1):171-178

26. Frey N, Olson EN. Cardiac hypertrophy: the good, the bad, and the ugly. Annu Rev Physiol. 2003;65:45-79.

27. Gruver CL, DeMayo F, Goldstein MA, Means AR. Targeted developmental overexpression of calmodulin induces proliferative and hypertrophic growth of cardiomyocytes in transgenic mice. Endocrinology. 1993;133(1):376-388.

28. Obata K, et al. Overexpression of calmodulin induces cardiac hypertrophy by a calcineurin-dependent pathway. Biochem Biophys Res Commun. 2005;338(2):1299-1305.

29. Oda T, et al. Nuclear translocation of calmodulin in pathological cardiac hypertrophy originates from ryanodine receptor bound calmodulin. J Mol Cell Cardiol. 2018;125:87-97.

30. Yamaguchi N, Chakraborty A, Pasek DA, Molkentin JD, Meissner G. Dysfunctional ryanodine receptor and cardiac hypertrophy: role of signaling molecules. Am J Physiol Heart Circ Physiol. 2011;300(6):H2187-H2195.

31. Loaiza R, et al. Heterogeneity of ryanodine receptor dysfunction in a mouse model of catecholaminergic polymorphic ventricular tachycardia. Circ Res. 2013;112(2):298-308.

32. Alvarado FJ, Valdivia CR. Ryanodine Receptor Mutations: In Sickness and in Health. Physiological Mini Reviews. 2018;11(3):23-35

33. Jabbari J, et al. New exome data question the pathogenicity of genetic variants previously associated with catecholaminergic polymorphic ventricular tachycardia. Circ Cardiovasc Genet. 2013;6(5):481-489.

34. Camors E, Valdivia HH. CaMKII regulation of cardiac ryanodine receptors and inositol triphosphate receptors. Front Pharmacol. 2014;5:101.

35. Wangüemert F, et al. Clinical and molecular characterization of a cardiac ryanodine receptor founder mutation causing catecholaminergic polymorphic ventricular tachycardia. Heart Rhythm. 2015;12(7):1636-1643.

36. Zhao YT, et al. Arrhythmogenesis in a catecholaminergic polymorphic ventricular tachycardia mutation that depresses ryanodine receptor function. Proc Natl Acad Sci USA. 2015;112(13):E1669-E1677.

37. Helms AS, et al. Sarcomere mutation-specific expression patterns in human hypertrophic cardiomyopathy. Circ Cardiovasc Genet. 2014;7(4):434-443.

38. McConnell BK, et al. Dilated cardiomyopathy in homozygous myosin-binding protein-C mutant mice. J Clin Invest. 1999;104(9):1235-1244.

39. Harris SP, et al. Hypertrophic cardiomyopathy in cardiac myosin binding protein-C knockout mice. Circ Res. 2002;90(5):594-601.

40. Chen PP, Patel JR, Powers PA, Fitzsimons DP, Moss RL. Dissociation of structural and functional phenotypes in cardiac myosin-binding protein C conditional knockout mice. Circulation. 2012;126(10):1194-1205.

41. Doetschman T. Influence of genetic background on genetically engineered mouse phenotypes. Methods Mol Biol. 2009;530:423-433

42. Helms AS, et al. Genotype-Dependent and -Independent Calcium Signaling Dysregulation in Human Hypertrophic Cardiomyopathy. Circulation. 2016;134(22):1738-1748.

43. Geisterfer-Lowrance AA, et al. A molecular basis for familial hypertrophic cardiomyopathy: a beta cardiac myosin heavy chain gene missense mutation. Cell. 1990;62(5):999-1006.

44. Helms AS, Day SM. Hypertrophic cardiomyopathy: single gene disease or complex trait? Eur Heart J. 2016;37(23):1823-1825.

45. Helms AS, Day SM. Other side of the coin: the missing heritability in hypertrophic cardiomyopathy. Eur Heart $J$. 2017;38(46):3469-3471.

46. Maron BJ, Ho CY. Hypertrophic cardiomyopathy without hypertrophy: an emerging pre-clinical subgroup composed of genetically affected family members. JACC Cardiovasc Imaging. 2009;2(1):65-68.

47. Maron BJ, et al. Development of left ventricular hypertrophy in adults in hypertrophic cardiomyopathy caused by cardiac myosin-binding protein C gene mutations. J Am Coll Cardiol. 2001;38(2):315-321.

48. Niimura $\mathrm{H}$, et al. Mutations in the gene for cardiac myosin-binding protein $\mathrm{C}$ and late-onset familial hypertrophic cardiomyopathy. NEngl J Med. 1998;338(18):1248-1257.

49. Van Driest SL, et al. Myosin binding protein C mutations and compound heterozygosity in hypertrophic cardiomyopathy. $J A m$ Coll Cardiol. 2004;44(9):1903-1910.

50. Girolami F, et al. Clinical features and outcome of hypertrophic cardiomyopathy associated with triple sarcomere protein gene mutations. J Am Coll Cardiol. 2010;55(14):1444-1453.

51. Emsley P, Lohkamp B, Scott WG, Cowtan K. Features and development of Coot. Acta Crystallogr D Biol Crystallogr. 2010;66(Pt 4):486-501.

52. Adams PD, et al. PHENIX: a comprehensive Python-based system for macromolecular structure solution. Acta Crystallogr D Biol Crystallogr. 2010;66(Pt 2):213-221.

53. Federico M, et al. Calcium-calmodulin-dependent protein kinase mediates the intracellular signalling pathways of cardiac apoptosis in mice with impaired glucose tolerance. J Physiol (Lond). 2017;595(12):4089-4108.

54. Palmer AE, Jin C, Reed JC, Tsien RY. Bcl-2-mediated alterations in endoplasmic reticulum Ca2+ analyzed with an improved genetically encoded fluorescent sensor. Proc Natl Acad Sci USA. 2004;101(50):17404-17409.

55. Liu P, Jenkins NA, Copeland NG. A highly efficient recombineering-based method for generating conditional knockout mutations. Genome Res. 2003;13(3):476-484.

56. Copeland NG, Jenkins NA, Court DL. Recombineering: a powerful new tool for mouse functional genomics. Nat Rev Genet. 2001;2(10):769-779

57. Cadiñanos J, Bradley A. Generation of an inducible and optimized piggyBac transposon system. Nucleic Acids Res. 
2007;35(12):e87.

58. Wong GT. Speed congenics: applications for transgenic and knock-out mouse strains. Neuropeptides. 2002;36(2-3):230-236.

59. Alvarado FJ, Chen X, Valdivia HH. Ablation of the cardiac ryanodine receptor phospho-site Ser2808 does not alter the adrenergic response or the progression to heart failure in mice. Elimination of the genetic background as critical variable. J Mol Cell Cardiol. 2017;103:40-47.

60. Benkusky NA, et al. Intact beta-adrenergic response and unmodified progression toward heart failure in mice with genetic ablation of a major protein kinase A phosphorylation site in the cardiac ryanodine receptor. Circ Res. 2007;101(8):819-829.

61. Chen $X$, et al. Sorcin ablation plus $\beta$-adrenergic stimulation generate an arrhythmogenic substrate in mouse ventricular myocytes. J Mol Cell Cardiol. 2018;114:199-210. 